\title{
SUBSEQUENCES OF ZEROS FOR BERNSTEIN SPACES AND THE COMPLETENESS OF SYSTEMS OF EXPONENTIALS IN SPACES OF FUNCTIONS ON AN INTERVAL
}

\author{
B. N. KHABIBULLIN, G. R. TALIPOVA, AND F. B. KHABIBULLIN
}

\begin{abstract}
Let $\sigma>0$. The symbol $B_{\sigma}^{\infty}$ denotes the space of all entire functions of exponential type not exceeding $\sigma$ that are bounded on the real axis. Various exact descriptions of uniqueness sequences for the Bernstein spaces $B_{\sigma}^{\infty}$ are given in terms of $\sigma$ and the Poisson and Hilbert transformations. These descriptions lead to completeness criteria for systems of exponentials (up to one or two members) in various classical function spaces on an interval (closed or open) of length $d$.
\end{abstract}

\section{§1. INTRODUCTION}

1.1. Main definitions, notions, and notation. We use the notation $\mathbb{C}_{+}:=\{z \in \mathbb{C}$ : $\operatorname{Im} z>0\}$ and $\mathbb{C}_{-}:=\{z \in \mathbb{C}: \operatorname{Im} z<0\}$ for the open upper and lower half-planes of the complex plane $\mathbb{C}$, and put $\mathbb{C}_{ \pm}:=\mathbb{C}_{-} \cup \mathbb{C}_{+}=\mathbb{C} \backslash \mathbb{R}$, where $\mathbb{R}$ is the real axis. The symbol $\mathbb{C}_{\infty}:=\mathbb{C} \cup\{\infty\}$ denotes the Riemann sphere; $\mathbb{D}:=\{z \in \mathbb{C}:|z|<1\}$ is the open unit disk centered at zero. Also, we use the symbols $\mathbb{R}_{*}:=\mathbb{R} \backslash\{0\}, \mathbb{C}_{*}:=\mathbb{C} \backslash\{0\}$, and $\mathbb{D}_{*}:=\mathbb{D} \backslash\{0\}$ to denote the "punctured" real axis, complex plane, and unit disk $\mathbb{D}$, respectively.

For a set $S \subset \mathbb{C}_{\infty}$, we denote by $\bar{S}$ and $\partial S$ the closure and the boundary of $S$ in $\mathbb{C}_{\infty}$, but for $S \subset \mathbb{R}$ the boundary of $S$ in $\mathbb{R}$ is denoted by $\partial_{\mathbb{R}} S$.

The symbol $I_{d} \subset \mathbb{R}$ (respectively, $\bar{I}_{d}$ ) will denote an open (respectively, closed) interval of length $d$.

Next, we denote by $\operatorname{Hol}(\Omega), \operatorname{sbh}(\Omega)$ and $\operatorname{har}(\Omega)$ the classes of holomorphic, subharmonic, and harmonic functions (respectively) in a domain $\Omega \subset \mathbb{C}_{\infty}$.

Let

$$
\Lambda=\left\{\lambda_{k}\right\}, \quad k=1,2, \ldots,
$$

be an at most countable sequence in a domain $\Omega \subset \mathbb{C}$ without limit points inside $\Omega$. Some points among the $\lambda_{k}$ may occur more than once. It is also permitted that $\Lambda$ is the empty set. With each sequence $\Lambda$, we associate the nonnegative integer-valued counting measure $n_{\Lambda}$ on $\Omega$ (the divisor) defined by

$$
n_{\Lambda}(S):=\sum_{\lambda_{k} \in S} 1, \quad S \subset \Omega,
$$

i.e., its value is the number of the $\lambda_{k}$ 's contained in $S$. For a point $z \in \mathbb{C}$, we put $n_{\Lambda}(z):=n_{\Lambda}(\{z\})$. We also introduce the radial counting function of the sequence $\Lambda \subset \mathbb{C}$

2010 Mathematics Subject Classification. Primary 30B50; Secondary 42C30.

Key words and phrases. Entire function, Bernstein space, sequence of uniqueness, completeness of exponentials, Poisson integral, Hilbert transformation.

Supported by RFBR (grant no. 13-01-00030a) and by the Federal targeted program "Scientific and pedagogical staff of innovative Russia" (contract no. 14.B37.21.0358). 
by the formula $n_{\Lambda}^{\mathrm{rad}}(t):=n(t \overline{\mathbb{D}}), t \geq 0$. In general, operations on sequences, relations involving them, and many other notions are the same as in 1 .

With a sequence $\Lambda \subset \mathbb{C}$, we associate the system of (multiple) exponentials

$$
\operatorname{Exp}^{\Lambda}:=\left\{z \mapsto z^{p-1} e^{i \lambda z}: z \in \mathbb{C}, \lambda \in \Lambda, 1 \leq p \leq n_{\Lambda}(\lambda), p \in \mathbb{N}\right\}
$$

where $\mathbb{N}$ is the set of positive integers. The imaginary unit $i$ arises in (1.3) mainly by tradition, which is related to completeness problems for systems of exponentials in spaces on subsets of the real axis (see [2, 3, 4, 5]), compare with [1]), and to the use of Fourier transforms. As usual, by a system $\operatorname{Exp}^{\Lambda}$ on a subset $S \subset \mathbb{C}$ we mean the system of restrictions of the functions in (1.3) to $S$.

Let $f \in \operatorname{Hol}(\Omega)$, and let $f \not \equiv 0$ on $\Omega$. The sequence of zeros of $f$ in $\Omega$ enumerated with multiplicities will be denoted by Zero $f$. A sequence $\Lambda$ is called a sequence of zeros for a subclass $H \subset \operatorname{Hol}(\Omega)$ if there exists a function $f \in H$ such that $\Lambda=Z_{\text {Zero }}$, i.e., $n_{\Lambda}=n_{\text {Zero }_{f}}$. A function $f \in \operatorname{Hol}(\Omega)$ vanishes on $\Lambda$ if $\Lambda \subset$ Zero $f_{f}$, i.e., $n_{\Lambda} \leq n_{\text {Zero }_{f}}$. A sequence $\Lambda$ is a subsequence of zeros for a subclass $H \subset \operatorname{Hol}(\Omega)$ if there exists a nonzero function $f \in H$ that vanishes on $\Lambda$. If the class $H$ is closed under subtraction, then a subsequence of zeros for $H$ is also called a nonuniqueness sequence, and any $\Lambda \subset \Omega$ that is not a subsequence of zeros for $H$ is also called a uniqueness sequence for $H$.

Let $\sigma>0$. The principal "model" class $H$ considered in the paper is the Bernstein space $B_{\sigma}^{\infty}$ (see [2]) of all entire functions $f \in \operatorname{Hol}(\mathbb{C})$ of exponential type at most $\sigma$ (i.e., $\left.\limsup \sup _{z \rightarrow \infty}(\log |f(z)|) /|z| \leq \sigma\right)$ bounded on the real axis, i.e., $\sup _{x \in \mathbb{R}}|f(x)|<+\infty$.

Alternatively (see [2]), the Bernstein space $B_{\sigma}^{\infty}$ can be defined as the class of all entire functions subject to the restriction

$$
\log |f(z)| \leq \sigma|\operatorname{Im} z|+c_{f}, \quad z \in \mathbb{C},
$$

where $c_{f}$ is a constant.

1.2. Some classical and some known results. Because of the well-known relationship between the completeness of systems of exponentials (1.3) in function spaces on an interval $I_{d}$ or a segment $\bar{I}_{d}$ and uniqueness sequences in the Bernstein space $B_{d / 2}^{\infty}$, some results will be stated as conditions for the (non)completeness of a systems of exponentials. It should be noted that many theorems in this spirit were proved or stated in [1, 2, 3, 4, 5, 6, 7, 8, 9. We formulate and discuss some of them.

Cartwright theorem. Suppose that for a sequence (1.1) the series

$$
\sum_{\operatorname{Im} \lambda_{k} \neq 0}\left|\operatorname{Im} \frac{1}{t-\lambda_{k}}\right|
$$

diverges for some $t \in \mathbb{R}$. Then $\Lambda$ is a uniqueness sequence for $B_{\sigma}^{\infty}$ for every $\sigma>0$, and the system $\operatorname{Exp}^{\Lambda}$ is complete in the spaces $C\left(\bar{I}_{d}\right)$ and $L^{p}\left(I_{d}\right)$ for any finite $d>0$ and $p \geq 1$. Furthermore, if the series

$$
\sum_{\operatorname{Im} \lambda_{k} \neq 0} \frac{1}{\left|\lambda_{k}\right|^{2}}
$$

converges, then the convergence of the series (1.5) for some particular $t$ implies its convergence for all $t \in \mathbb{R}$.

Remark 1. The divergence of the series (1.6) and even of the series $\sum_{\lambda_{k} \neq 0} 1 /\left|\lambda_{k}\right|^{\alpha}$ for some $\alpha>1$, and also the condition that either the upper density

$$
\limsup _{t \rightarrow+\infty} \frac{n_{\Lambda}^{\mathrm{rad}}(t)}{t}
$$


or the averaged upper density $(0 \notin \Lambda)$

$$
\limsup _{r \rightarrow+\infty} \frac{N_{\Lambda}(r)}{r}, \quad N_{\Lambda}(r):=\int_{0}^{r} \frac{n_{\Lambda}^{\operatorname{rad}}(t)}{t} d t=\sum_{k} \log ^{+} \frac{\left|\lambda_{k}\right|}{r},
$$

is infinite have the same consequence as the divergence of the series (1.5) in the Cartwright theorem, see [5, Theorem 7].

Remark 2. For a wide class of spaces including $B_{\sigma}^{\infty}$, the sequence $\Lambda$ remains to be a (non)uniqueness sequence after a shift of a finite number of points, and the (non)completeness of a sequence of exponentials $\operatorname{Exp}^{\Lambda}$ is stable under this transformation in the function spaces under study (see [5] and a general result in [1, Theorem 1.1.4]). Therefore, without loss of generality we may assume if necessary that $0 \notin \Lambda$ for the sequence (1.1).

We present a weak converse to the Cartwright theorem.

Schwartz theorem (see [4] and [5, Theorem 41]). Suppose that a sequence (1.1) satisfies the following two conditions:

$$
0<\alpha \leq\left|\arg \lambda_{k}\right| \leq \pi-\alpha, \quad k \in \mathbb{N}, \quad \sum_{\lambda_{k} \neq 0}\left|\operatorname{Im} \frac{1}{\lambda_{k}}\right|<+\infty,
$$

or only the condition $\sum_{\lambda_{k} \neq 0} 1 /\left|\lambda_{k}\right|<+\infty$. Then $\Lambda$ is a subsequence of zeros for the spaces $B_{\sigma}^{\infty}$ for all $\sigma>0$, and the system $\operatorname{Exp}^{\Lambda}$ is not complete in the spaces $C\left(\bar{I}_{d}\right)$ and $L^{p}\left(I_{d}\right)$ for every $d>0$.

New proofs of the Cartwright and Schwartz theorems will be presented below to illustrate the specific features of our approach.

The above theorems have a drawback. Specifically, they do not "feel" the removal from $\Lambda$ or the addition to $\Lambda$ of finitely many points. To a certain extent, this is overcome in Levinson's theorems, see [3]. We state only one to illustrate them.

Levinson theorem. Suppose that $p \in[1,+\infty]$, and the sequence (1.1) has the property that the characteristic

$$
\limsup _{r \rightarrow \infty}\left(N_{\Lambda}(r)-\frac{d}{\pi} r+\frac{\log r}{p}\right)
$$

is equal to $+\infty$ for $p=1,+\infty$ or is not equal to $-\infty$ for $1<p<+\infty$. Then the system $\operatorname{Exp}^{\Lambda}$ is complete in $L^{p}\left(I_{d}\right)$ for $p \in[1,+\infty)$ and in $C\left(\bar{I}_{d}\right)$ for $p=+\infty$. In (1.10), the number $p$ cannot be replaced by a smaller one.

The most substantial generalization of the Levinson theorem was published recently in a joint paper by Makarov and Poltoratskiu in [10, §2.11]. In that paper, some general criteria were proved (see [10, §3.2, Proposition, Theorem, and Example]), which describe the nonuniqueness sets for a wide class of model spaces. Later, these criteria were generalized by Baranov, see [12, Theorems 9.1.1 and 9.1.2]. A certain criterion for a sequence to be one of uniqueness for a Bernstein space can also be deduced from those results. We do not state it here for two reasons. First, it requires some preparations. Second, though the Hilbert transformation is employed both in the results by Makarov, Poltoratskii, and Baranov and in our main theorem, this is done in opposite ways in a sense. In the Makarov-Poltoratskii-Baranov theorems, it is required to construct (or to prove the existence of) certain special functions for the given sequence $\Lambda$ and the space in question; the Hilbert transform of one of them must possess certain specific properties. But under our approach, the verification of an infinite series of uniform integral estimates is required for a special class of test functions that are defined in terms of the Hilbert transformation. 
The following classical theorem stays until now a deepest result on the completeness of systems of exponentials and on (non)uniqueness sequences for the Bernstein spaces.

Beurling-Malliavin theorem ([5, Theorem 77], Redheffer's interpretation). Suppose that $\lambda_{k} \neq 0$ in the sequence (1.1). If there exists a number $c>0$ and a sequence $\left\{n_{k}\right\}_{k \in \mathbb{N}}$ of pairwise different integers such that the series $\sum_{k \in \mathbb{N}}\left|\frac{1}{\lambda_{k}}-\frac{c}{2 \pi n_{k}}\right|$ converges, then $\Lambda$ is a subsequence of zeros for $B_{d / 2}^{\infty}$ and the system $\operatorname{Exp}^{\Lambda}$ is not complete in $C\left(\bar{I}_{d}\right)$ and in $L^{p}\left(I_{d}\right), p \geq 1$, whenever $d>c$. Conversely, if this series diverges for every sequence $\left\{n_{k}\right\}_{k \in \mathbb{N}}$ of pairwise different integers, then $\Lambda$ is a uniqueness sequence for $B_{d / 2}^{\infty}$ and the system $\operatorname{Exp}^{\Lambda}$ is complete in $C\left(\bar{I}_{d}\right)$ and in $L^{p}\left(I_{d}\right)$ for every $d<c$.

The property to be a (non)uniqueness sequence as well as the property to be a (non)complete sequence of exponentials may happen to be stable under small variations of the sequence (respectively, under small shifts of the exponents $\Lambda$ in (1.3)). Each stability statement of this sort yields immediately conditions sufficient for (non)uniqueness or (non)completeness if we start with a sequence $\Lambda$ for which the required property is already known. The following statement is the origin of many such results.

Redheffer-Aleksander theorem. If two sequences

$$
\Lambda:=\left\{\lambda_{k}\right\}, \quad \Gamma:=\left\{\gamma_{k}\right\}, \quad k=1,2, \ldots,
$$

satisfy the condition $\sum_{k=1}^{\infty} \frac{\left|\lambda_{k}-\gamma_{k}\right|}{1+\left|\operatorname{Im} \lambda_{k}\right|+\left|\operatorname{Im} \gamma_{k}\right|}<\infty$, then, for any $d>0$, the systems $\operatorname{Exp}^{\Lambda}$ and $\operatorname{Exp}^{\Gamma}$ are simultaneously complete or not in each of the spaces $C\left(\bar{I}_{d}\right)$ and $L^{p}\left(I_{d}\right)$, $1 \leq p<\infty$.

The following theorem was obtained by Baranov not very long ago.

Baranov theorem (see [11, Theorem 2.3], 12, Theorem 9.2.1]). If a sequence (1.11) in the upper half-plane $\mathbb{C}_{+}$satisfies the condition $\sup _{t \in \mathbb{R}} \sum_{k} \frac{\left|\lambda_{k}-\gamma_{k}\right|}{\left|t-\lambda_{k}-i\right|}<\infty$, then $\left\{\lambda_{k}\right\}$ and $\left\{\gamma_{k}\right\}$ may be nonuniqueness sequences for $B_{\sigma}^{\infty}$ only simultaneously.

Baranov's theorem is also true for the Paley-Wiener spaces $P W_{\sigma}^{p}$.

\section{§2. Principal Results}

2.1. Criterion for being a subsequence of zeros for Bernstein spaces. Recall that, for a function $\varphi \in L^{1}(\mathbb{R}), \varphi: \mathbb{R}_{*} \rightarrow \mathbb{R}$, its direct Hilbert transform $\mathrm{H}$ is defined by the integral (see [13, 14, 15])

$$
(\mathrm{H} \varphi)(x):=\frac{1}{\pi} \int_{\mathbb{R}} \frac{\varphi(t)}{x-t} d t, \quad x \in \mathbb{R}_{*},
$$

where the dashed integral denotes the Cauchy principal value. This function $\mathrm{H} \varphi$ is defined a.e. on $\mathbb{R}$. The inverse Hilbert transform differs 1 only by the sign:

$$
\left(\mathrm{H}^{-1} \varphi\right)(x):=\frac{1}{\pi} f_{\mathbb{R}} \frac{\varphi(t)}{t-x} d t=-(\mathrm{H} \varphi)(x), \quad x \in \mathbb{R}_{*} .
$$

Classes $R \mathcal{P}_{0}^{m}$ of test functions. In what follows, we only consider the case where

$$
m \in(\mathbb{N} \backslash\{1\}) \cup\{\infty\} .
$$

\footnotetext{
${ }^{1}$ Sometimes, especially in applications to engineering, the terms are used in the opposite sense.
} 
Definition 1. We introduce the class $R \mathcal{P}_{0}^{m}$ of test functions as the subclass of all positive continuous functions

(2.3.

(2.3t)

(2.3p)

$$
\varphi: \mathbb{R}_{*} \rightarrow[0,+\infty), \quad \varphi \in C\left(\mathbb{R}_{*}\right),
$$

with the zero set $Z_{\varphi}:=\left\{x \in \mathbb{R}_{*}: \varphi(x)=0\right\}$

the restriction of $\varphi$ to $\mathbb{R} \backslash\left(\{0\} \cup Z_{\varphi} \cup E_{\varphi}\right)=\mathbb{R}_{*} \backslash\left(Z_{\varphi} \cup E_{\varphi}\right)$ belongs to the class

$$
C^{m}\left(\mathbb{R} \backslash\left(\{0\} \cup Z_{\varphi} \cup E_{\varphi}\right)\right)=C^{m}\left(\mathbb{R}_{*} \backslash\left(Z_{\varphi} \cup E_{\varphi}\right)\right),
$$

and the following conditions are fulfilled:

- the compact support requirement

$$
\varphi(x) \equiv 0, \quad|x| \geq R_{\varphi}>0,
$$

where $R_{\varphi}>0$ is a constant depending on $\varphi$;

- seminormalization at zero

$$
\limsup _{0 \neq x \rightarrow 0} \frac{\varphi(x)}{-\log |x|} \leq 1
$$

- conjugate positivity condition

$$
(-\mathrm{H} \varphi)^{\prime}(x)=\frac{1}{\pi} f_{\mathbb{R}} \frac{\varphi(t)-\varphi(x)}{(t-x)^{2}} d t \geq 0, \quad x \in \mathbb{R}_{*} \backslash\left(Z_{\varphi} \cup E_{\varphi}\right) ;
$$

after a change of variables, this inequality can be rewritten in the form

$$
(-\mathrm{H} \varphi)^{\prime}(x)=\frac{1}{\pi} \int_{0}^{+\infty} \frac{\varphi(x+t)+\varphi(x-t)-2 \varphi(x)}{t^{2}} d t \geq 0
$$

next, equality in (2.6) -(2.7) is ensured by the last identity in (4.7) (see the final part of Proposition 2 proved below).

By (2.1) and (2.6), the conjugate positivity conditions (2.6) -(2.7) are equivalent to the requirement that the inverse Hilbert transform $\mathrm{H}^{-1} \varphi=-\mathrm{H} \varphi$ be monotone increasing $2^{2}$ on each connected component of the complement to $\mathbb{R}_{*}$ of the set $Z_{\varphi} \cup E_{\varphi}$. Since this set is closed in $\mathbb{R}_{*}$, all connected components mentioned above are intervals.

The class $R \mathcal{P}_{0}^{m}$ of test functions is invariant under homothety, which will be useful in the sequel (see 86 ): if $\varphi$ belongs to $R \mathcal{P}_{0}^{m}$, then for every $r \in \mathbb{R}_{*}$ the function

$$
\varphi(\cdot / r): t \mapsto \varphi(t / r), \quad t \in \mathbb{R}_{*}, \quad \text { also belongs to } R \mathcal{P}_{0}^{m} .
$$

The left-hand sides of (2.6) -2.7) can be replaced by the direct Hilbert transform of the derivative $\varphi^{\prime}$ because $(-\mathrm{H} \varphi)^{\prime}=-\mathrm{H} \varphi^{\prime}=H^{-1} \varphi^{\prime}$, provided $\varphi^{\prime}$ belongs to $L^{p}(\mathbb{R})$ with some $p>1$ (see [13, Subsection 4.8]), or provided we treat the derivatives and Hilbert transforms in the sense of the theory of distributions (see [15, §3.3]).

Poisson transformation. For $\lambda \in \mathbb{C}_{ \pm}:=\mathbb{C} \backslash \mathbb{R}$, the Poisson integral $\mathrm{P}_{\mathbb{C}_{ \pm}} \varphi$ of a function $\varphi \in L^{1}(\mathbb{R})$ is defined by

$$
\left(\mathrm{P}_{\mathbb{C}_{ \pm}} \varphi\right)(\lambda):=\frac{1}{\pi} \int_{-\infty}^{+\infty} \frac{|\operatorname{Im} \lambda|}{(t-\operatorname{Re} \lambda)^{2}+(\operatorname{Im} \lambda)^{2}} \varphi(t) d t, \quad \operatorname{Im} \lambda \neq 0 .
$$

For $\lambda \in \mathbb{R}_{*}$, we put

$$
\left(\mathrm{P}_{\mathbb{C}_{ \pm}} \varphi\right)(\lambda):=\varphi(\lambda), \quad \lambda \in \mathbb{R}_{*} .
$$

The Poisson integral in (2.9) is a harmonic function on $\mathbb{C} \backslash \mathbb{R}$. Taken together, the definitions (2.9) and (2.10) determine the Poisson transformation $\mathrm{P}_{\mathbb{C}_{ \pm}}$.

\footnotetext{
${ }^{2}$ A function $f$ is monotone increasing on $I \subset \mathbb{R}$ if $f\left(x_{1}\right) \leq f\left(x_{2}\right)$ whenever $x_{1} \leq x_{2}, x_{1}, x_{2} \in I$.
} 
Theorem 1 (on uniqueness sequences for the Bernstein spaces). Let $\Lambda=\left\{\lambda_{k}\right\}$ be a sequence in $\mathbb{C}$ with $0 \notin \Lambda$, and let $\sigma \in(0,+\infty)$. The following three statements are equivalent:

1) $\Lambda=\left\{\lambda_{k}\right\}_{k \in \mathbb{N}}$ is a uniqueness sequence for the Bernstein space $B_{\sigma}^{\infty}$;

2) for some (for arbitrary) $m$ in (2.2) we have

$$
\sup _{\varphi \in R \mathcal{P}_{0}^{m}}\left(\sum_{k \in \mathbb{N}}\left(\mathrm{P}_{\mathbb{C}_{ \pm}} \varphi\right)\left(\lambda_{k}\right)-\frac{\sigma}{\pi} \int_{-\infty}^{+\infty} \varphi(t) d t\right)=+\infty ;
$$

3) condition (2.11) is fulfilled with the replacement of the supremum over $R \mathcal{P}_{0}^{m}$ by the supremum over the smaller class of smooth test functions $R \mathcal{P}_{0}^{\infty} \cap C^{\infty}\left(\mathbb{R}_{*}\right)$.

Remark 2 in Subsection 1.2 shows that the condition $0 \notin \Lambda$ does not lead to any loss of generality.

Remark 3. If $\Lambda \subset \mathbb{R}_{*}$ is a real sequence, then (2.11) becomes very simple:

$$
\sup _{\varphi \in R \mathcal{P}_{0}^{m}}\left(\sum_{\lambda \in \Lambda} \varphi(\lambda)-\frac{\sigma}{\pi} \int_{-\infty}^{+\infty} \varphi(t) d t\right)=+\infty .
$$

Remark 4. As was mentioned in Remark 1 (see Subsection 1.2), Theorem 1 is interesting only in the case where the series (1.5) converges for some $t \in \mathbb{R}$, and the sequence $\Lambda$ has finite upper density. Note that every sequence $\Lambda$ of finite upper density has convergence exponent of at most 1 , which means the convergence of every series $\sum_{\lambda_{k} \neq 0} 1 /\left|\lambda_{k}\right|^{\alpha}$ for every $\alpha>1$. In particular, the series (1.6) converges. Then the series $\sum_{\operatorname{Im} \lambda_{k} \neq 0}\left|\operatorname{Im} \frac{1}{\lambda_{k}}\right|$. also converges. Moreover, the series (1.5) converges uniformly in $t \in[-r, r] \subset \mathbb{R}$ for every fixed $r>0$. Indeed, let $t \in[-r, r]$. Then, for every $R^{\prime}>R \geq 2 r$, it is easy to deduce the inequality

$$
\sum_{\substack{\operatorname{Im} \lambda_{k} \neq 0 \\ R \leq\left|\lambda_{k}\right| \leq R^{\prime}}}\left|\operatorname{Im} \frac{1}{t-\lambda_{k}}\right| \leq \sum_{\substack{\operatorname{Im} \lambda_{k} \neq 0 \\ b R \leq\left|\lambda_{k}\right| \leq R^{\prime}}}\left|\operatorname{Im} \frac{1}{\lambda_{k}}\right|+\sum_{R \leq\left|\lambda_{k}\right| \leq R^{\prime}} \frac{2 r}{\left|\lambda_{k}\right|^{2}},
$$

where the right-hand side tends to zero as $R^{\prime}, R \rightarrow+\infty$ independently of $t \in[-r, r]$. Now, the uniform convergence follows from the Cauchy criterion. Since every test function has compact support (included in $\left.\left[-R_{\varphi}, R_{\varphi}\right]\right)$, in the sum $\sum_{k \in \mathbb{N}}\left(\mathrm{P}_{\mathbb{C}_{ \pm}} \varphi\right)\left(\lambda_{k}\right)$ we can interchange summation and the (Poisson) integral 3 Consequently, if the series (1.5) converge (for some $t$ ) and we have (1.6), then

$$
\sum_{k \in \mathbb{N}}\left(\mathrm{P}_{\mathbb{C}_{ \pm}} \varphi\right)\left(\lambda_{k}\right)=\int_{\mathbb{R}}\left(\sum_{\operatorname{Im} \lambda_{k} \neq 0} \frac{1}{\pi}\left|\operatorname{Im} \frac{1}{t-\lambda_{k}}\right|\right) \varphi(t) d t+\sum_{\operatorname{Im} \lambda_{k}=0} \varphi\left(\lambda_{k}\right)
$$

so that the sum in (2.11) can be replaced by the right-hand side of the last identity.

Remark 5. In conditions (2.11) and (2.12), a direct analogy can be observed with the definitions of the natural order relations for real Radon measures or for distributions. Indeed, if $\nu$ and $\mu$ are two such measures or distributions on $\mathbb{C}$, then, by definition, $\nu \leq \mu$ means that

$$
\sup _{\varphi \in\left(C_{0}^{\infty}(\mathbb{C})\right)^{+}}(\nu(\varphi)-\mu(\varphi)) \leq 0
$$

where $\left(C_{0}^{\infty}(\mathbb{C})\right)^{+}$is the class of all compactly supported infinitely differentiable positive functions on $\mathbb{C}$.

\footnotetext{
${ }^{3}$ The possibility of this interchange also follows from the positivity of the terms of the series, see 16 Chapter IV, §4, Corollary 4].
} 
On the other hand, every sequence $\Lambda=\left\{\lambda_{k}\right\}_{k \in \mathbb{N}} \subset \mathbb{C}$ of points gives rise to the counting measure $n_{\Lambda}$ defined by the rule (1.2). The measure $\mu_{\sigma}$ on $\mathbb{R}$ with the density $d \mu_{\sigma}(x):=\frac{\sigma}{\pi} d x, x \in \mathbb{R}$, is the Riesz measure of the subharmonic function $M_{\sigma}(z):=$ $\sigma|\operatorname{Im} z|, z \in \mathbb{C}, \mu_{\sigma}:=\frac{1}{2 \pi} \Delta M_{\sigma} \geq 0$, where $\Delta$ stands for the Laplace operator on Schwatrz distributions. The function $M_{\sigma}$ occurs on the right in (1.4) and determines the Bernstein class $B_{\sigma}^{\infty}$. In this notation, (2.11) and (2.12) can be rewritten in the form

$$
\sup _{\varphi \in R \mathcal{P}_{0}^{m}}\left(n_{\Lambda}\left(\mathrm{P}_{\mathbb{C}_{ \pm}} \varphi\right)-\mu_{\sigma}(\varphi)\right)<+\infty .
$$

It is useful to compare this formula with relation (2.14). An analogy with that relation is fairly transparent, especially in item 3), where we use infinitely differentiable test functions belonging to $R \mathcal{P}_{0}^{\infty} \cap C^{\infty}\left(\mathbb{R}_{*}\right)$.

\subsection{Completeness with excess 0 or 1 for systems of exponentials in function} spaces on an interval. In this subsection, we employ the terminology and notation from [1] (sometimes with slight modifications). We recall some definitions. A system of vectors in a locally convex space $E$ is complete if the closure of its linear hull coincides with $E$. A system of vectors is minimal in $E$ if no vector in the system belongs to the closure of the linear hull of the other vectors. A system is said to be exact if it is complete and minimal. Let $\mathcal{E}_{\Lambda}$ be a sequence of pairwise different vectors in $\mathcal{E} \subset E$, and suppose that it is indexed by points of $\Lambda=\left\{\lambda_{k}\right\} \subset \mathbb{C}$. The system $\mathcal{E}_{\Lambda}$ is said to have an excess $\operatorname{exc} \mathcal{E}_{\Lambda}=q \in \mathbb{Z}$ in $E$ (relative to $\mathcal{E}$ ) if a system exact in $E$ arises

- after removal of $q$ vectors from $\mathcal{E}_{\Lambda}$ if $q \geq 0$;

- after addition of $|q|$ new vectors taken from $\mathcal{E}$ to $\mathcal{E}_{\Lambda}$ if $q<0$.

If completeness is not destroyed (does not arise) after removal (respectively, addition) of an arbitrary finite collection of pairwise different vectors from $\mathcal{E}_{\Lambda}$ (respectively, vectors belonging to $\mathcal{E} \backslash \mathcal{E}_{\Lambda}$ ), we put exc $\mathcal{E}_{\Lambda}:=+\infty$ (respectively, exc $\mathcal{E}_{\Lambda}:=-\infty$ ).

In the sequel, when talking about the excess of a system of exponentials $\operatorname{Exp}^{\Lambda}$ in a function space, we shall write $\operatorname{exc} \Lambda$ in place of $\operatorname{exc} \operatorname{Exp}^{\Lambda}$. For the spaces under study, the excess for multiple exponentials is well defined because it does not depend on the exponentials that are removed or added (general results on this matter can be found in [1, Theorem 1.1.4(3), Subsection 1.1.3]).

Theorem 2 (on the completeness of a system of exponentials on intervals). If a sequence $\Lambda \subset \mathbb{C}$ of points, $0 \notin \Lambda$, satisfies (2.11) or, under the assumption $\Lambda \subset \mathbb{R}$, it satisfies (2.12), then the system $\operatorname{Exp}^{\Lambda}$ is complete in any space $C\left(\bar{I}_{2 \sigma}\right)$ or $L^{p}\left(I_{2 \sigma}\right), p \geq 1$. Conversely, if the left-hand side in (2.11) or, under the restriction $\Lambda \subset \mathbb{R}$, in (2.12), is finite, then, relative to the system of all multiple exponentials, we have exc $\Lambda \leq 0$ for $C\left(\bar{I}_{2 \sigma}\right)$ and for $L^{p}\left(I_{2 \sigma}\right)$, where $p \geq 2$; moreover, exc $\Lambda \leq 1$ for $L^{p}\left(I_{2 \sigma}\right)$ provided $1 \leq p<2$.

Furthermore, in (2.11) or (2.12), the classes $R \mathcal{P}_{0}^{m}$ of test functions can be replaced (in any situation) by the smaller class that occurs in item 3) of Theorem 1.

Remark 6. In a somewhat different and simpler form, Theorems 1 and 2 were announced in the 3rd and 4th edition of the book [1, see Theorem 2.1.12 and Corollary 2.1.2 therein, and also in the short survey [17, Theorem 4 and Corollary 2]. It should also be noted that when we want to deduce from Theorem 1 various conditions sufficient for uniqueness (see Subsection 6.1 below) and also conditions of completeness for a system of exponentials, it is useful to possibly extend the set of test functions (see the implication 2 ) $\Longrightarrow 1$ ) in Theorem 1 and the first part of Theorem 21). Conversely, when we study conditions sufficient for nonuniqueness or for noncompleteness of systems of exponentials, it is convenient to possibly restrict the class of test functions without destruction of Theorem 1 
(see the implication 3) $\Longrightarrow 1$ )) or the second part of Theorem 2 on completeness (see the final statement in it). Thus, both extension and restriction of the class of test functions are of interest. At the same time, the problem of restriction of the class of test functions in these theorems is much subtler and much more complicated then the problem of extending the class $R \mathcal{P}_{0}^{2}$. We are going to return to this elsewhere.

\section{§3. Description of uniqueness SEQUEnCES FOR BERNSTEIN SPACES IN TERMS OF THE JENSEN POTENTIALS}

We shall need some definitions and facts from the paper [18 and the survey [1].

In the sequel, the symbol $\Subset$ denotes a precompact inclusion.

Definition 2. A function $V$ subharmonic in $\mathbb{C}_{*}$ is called a Jensen potentia 4 (with a pole at 0 ) if the following three conditions are fulfilled:

1) $V(\zeta) \geq 0$ for $\zeta \in \mathbb{C}_{*}$ (positivity);

2) there exists a compact set $K \Subset \mathbb{C}$ such that $V \equiv 0$ on $\mathbb{C} \backslash K$ (compact support);

3) $\lim \sup _{\zeta \rightarrow 0} \frac{V(\zeta)}{-\log |\zeta|} \leq 1$ (seminormalization at zero).

The class of all Jensen potentials will be denoted by $\mathcal{P}_{J_{0}}$.

In fact, the seminormalization condition for $V$ at zero gives more: there exists a number $R_{V}>0$ such that

$$
V(\zeta) \leq \log +\frac{R_{V}}{|\zeta|}, \quad \zeta \in \mathbb{C}_{*}
$$

(see [18, Proposition 1.5]), and if $V(\infty):=0$, then $V \in \operatorname{sbh}\left(\mathbb{C}_{\infty} \backslash\{0\}\right)$.

The role played by the Jensen potentials in the poof of Theorem 1 is dictated by a result proved in [18, Theorem 2]. Let $M \in \operatorname{sbh}(\mathbb{C})$ with $M \not \equiv-\infty$, and let $\nu_{M}:=\frac{1}{2 \pi} \Delta M$ be the Riesz measure for $M$, where $\Delta$ is the Laplace operator on distributions.

We introduce the weighted space of entire functions

$$
\operatorname{Hol}(\mathbb{C} ; M):=\left\{f \in \operatorname{Hol}(\mathbb{C}): \sup _{z \in \mathbb{C}} \frac{|f(z)|}{\exp M}<+\infty\right\}
$$

Theorem A (see [18, Introduction, Theorem 2]). Let $M \in \operatorname{sbh}(\mathbb{C})$. If there exists a constant $C \in \mathbb{R}$ such that

$$
\sup _{|z-\zeta| \leq 1} M(\zeta) \leq M(z)+C \text { for all } z \in \mathbb{C},
$$

then the following statements are equivalent:

1) the sequence $\Lambda=\left\{\lambda_{k}\right\} \subset \mathbb{C}$ is a uniqueness sequence for $\operatorname{Hol}(\mathbb{C} ; M)$;

2) we have

$$
\sup _{V \in \mathcal{P}_{J_{0}}}\left(\sum_{k} V\left(\lambda_{k}\right)-\int V d \nu_{M}\right)=+\infty .
$$

It has been known since long ago (see 2]) that the Bernstein space $B_{\sigma}^{\infty}$ coincides with $\operatorname{Hol}(\mathbb{C} ; M)$ for $M(z) \equiv \sigma|\operatorname{Im} z|, z \in \mathbb{C}$, and, clearly, such a subharmonic function $M$, whose Riesz measure's density is

$$
d \nu_{M}(t)=\frac{\sigma}{\pi} d t, \quad t \in \mathbb{R}
$$

(where $d t$ is the Lebesgue measure on $\mathbb{R}$ ), satisfies (3.1) with $C=\sigma$.

\footnotetext{
${ }^{4}$ In [18, 1] and other our papers the term "Jensen function" was used, but it turned out that it had been employed before in the theory of generalized analytic functions (see the references in [19]). This forced us to pass to the term "Jensen potential".
} 
Recall that the Jensen measures $\mu$ are defined as compactly supported nonnegative measures that satisfy the condition

$$
u(0) \leq \int u d \mu, \quad u \in \operatorname{sbh}(\mathbb{C}) .
$$

We denote this class of measures by $J_{0}$. The class of all Jensen potentials $\mathcal{P}_{J_{0}}$ coincides (see [18, §4, Proposition 4.1]) with the class of functions

$$
V_{\mu}(\zeta):=\int \log \left|1-\frac{z}{\zeta}\right| d \mu(z)=\int \log |\zeta-z| d \mu(z)-\log |\zeta|, \quad \mu \in J_{0} .
$$

By the proof of the main theorem in [18, §2, Main theorem $]$ and by [18, $\S 2$, Remark 1], in that main theorem we may restrict ourselves to smooth (with densities of class $C^{\infty}(\mathbb{C})$ ) Jensen measures supported outside an arbitrarily fixed disk $R \mathbb{D}$. By the definition (3.4), such measures give rise to Jensen potentials smooth in $\mathbb{C}_{*}$ and harmonic in $R \mathbb{D}_{*}$, see [18, $\S 4$, Example 2]. Theorem A is deduced from [18, §2, Main theorem] with the help of [18, $\S 5$, Theorem 2], where Jensen measures are replaced by Jensen potentials. Thus, also in Theorem A smooth Jensen potentials harmonic in $R \mathbb{D}_{*}$ would suffice. This yields the following statement.

Remark 7. In formula (3.2) (see Theorem A) the term $\sup _{V \in \mathcal{P}_{J_{0}}}$ can be replaced by the supremum over all infinitely differentiable Jensen potentials $V \in \mathcal{P}_{J_{0}} \cap C^{\infty}\left(\mathbb{C}_{*}\right)$ with harmonic restriction to $R \mathbb{D}_{*}$.

In the special case where $\nu_{1}=n_{\Lambda}, d \nu_{2}=\nu_{M}$, and $M(z) \equiv \sigma|\operatorname{Im} z|$, formula (3.3) and Remark 7 allow us to restate Theorem A as follows.

Theorem 3. Let $\Lambda=\left\{\lambda_{k}\right\}$ be a sequence in $\mathbb{C}$, and let $\sigma \in(0,+\infty)$. The following three statements are equivalent:

1) $\Lambda=\left\{\lambda_{k}\right\} \subset \mathbb{C}$ is a uniqueness sequence for the Bernstein space $B_{\sigma}^{\infty}$;

2) for some (for any) $R>0$ we have

$$
\sup \left\{\sum_{k} V\left(\lambda_{k}\right)-\frac{\sigma}{\pi} \int_{-\infty}^{+\infty} V(t) d t: V \in \mathcal{P}_{J_{0}} \cap C^{\infty}\left(\mathbb{C}_{*}\right),\left.V\right|_{R \mathbb{D}_{*}} \in \operatorname{har}\left(R \mathbb{D}_{*}\right)\right\}=+\infty
$$

3) the following condition holds true:

$$
\sup _{V \in \mathcal{P}_{J_{0}}}\left(\sum_{k} V\left(\lambda_{k}\right)-\frac{\sigma}{\pi} \int_{-\infty}^{+\infty} V(t) d t\right)=+\infty
$$

\section{§4. Extension of TEST FunCtions}

The following statement will play a principal role in this section.

Proposition 1. Suppose that $\mathcal{O}$ is an open subset of $\mathbb{C}$ and a function $V: \mathcal{O} \rightarrow \mathbb{R}$ is continuous on $\mathcal{O}, V \in \operatorname{har}(\mathcal{O} \backslash \mathbb{R})$, and

$$
V \in C^{1}\left(\overline{\mathbb{C}}_{+} \cap \mathcal{O}\right), \quad V \in C^{1}\left(\overline{\mathbb{C}}_{-} \cap \mathcal{O}\right) .
$$

The function $V$ is subharmonic on $\mathcal{O}$ if and only if

$$
\begin{array}{r}
\left(\frac{\partial V}{\partial \vec{n}_{+}^{\text {in }}}+\frac{\partial V}{\partial \vec{n}_{-}^{\text {in }}}\right)(x):=\lim _{0<y \rightarrow 0} \frac{V(x+i y)-V(x)}{y}+\lim _{0>y \rightarrow 0} \frac{V(x+i y)-V(x)}{-y} \geq 0, \\
x \in \mathbb{R} \cap \mathcal{O},
\end{array}
$$

\footnotetext{
${ }^{5}$ In the proof of that theorem, there are some typos in item $2 \mathrm{a}$ ). To fix them, it suffices to replace the product $\varphi_{\Lambda} g_{R}$ by $\varphi_{\Lambda} \exp g_{R}$ throughout. However, the same correction was already made in the more recent and informative statement [20, Proof of Proposition 9.2, item (a)].
} 
where $\frac{\partial}{\partial \vec{n}_{+}^{\text {in }}}$ and $\frac{\partial}{\partial \vec{n}_{-}^{\text {in }}}$ denote differentiations in the direction of the inner unit normal of $\mathbb{C}_{+} \cap \mathcal{O}$ and $\mathbb{C}_{-} \cap \mathcal{\mathcal { O }}$, respectively. Moreover, the density $\nu_{V}$ of the Riesz measure of the function $V \in \operatorname{sbh}(\mathcal{O})$ with support $\operatorname{supp} \nu_{V} \subset \mathbb{R} \cap \mathcal{O}$ coincides with the left-hand side of inequality (4.1) divided by $2 \pi$.

Moreover, if under condition (4.1) the function $V$ is also defined as positive and continuous in a neighborhood $\mathcal{O}_{0}$ (open in $\mathbb{C}$ ) of the union $A_{0}:=Z_{0} \cup E_{0}$ of two closed subsets $Z_{0}$ and $E_{0}$ of $\mathbb{R} \backslash \mathcal{O}$, is subharmonic on $\mathcal{O}_{0} \backslash A_{0}$, and satisfies $V(x) \equiv 0$ for $x \in Z_{0}$, and $E_{0}$ is a polar set in $\mathbb{C}$, then $V$ is subharmonic in the open neighborhood $\mathcal{O} \cup \mathcal{O}_{0}$ of $(\mathbb{R} \cap \mathcal{O}) \cup A_{0} \subset \mathbb{R}$.

Proof. Only the case where $\mathbb{R} \cap \mathcal{O} \neq \varnothing$ is nontrivial.

Lemma A. Let $D$ be a domain in $\mathbb{C}$, and let $S$ be a $C^{1}$-curve that divides $D$ in two subdomains $D_{1}$ and $D_{2}$.

Suppose that $V \in C_{\mathbb{R}}(D) \cap C^{2}\left(D_{1} \cup D_{2}\right)$ and $V \in \operatorname{sbh}\left(D_{k}\right), k=1,2$. If

$$
\left.V\right|_{D_{k} \cup S}=: V_{k} \in C^{1}\left(D_{k} \cup S\right), \quad k=1,2,
$$

then the function is subharmonic in $D$ if and only if

$$
\frac{\partial V_{1}}{\partial \vec{n}_{1}^{\text {in }}}+\frac{\partial V_{2}}{\partial \vec{n}_{2}^{\text {in }}} \geq 0
$$

on $S$, where the $\frac{\partial}{\partial \vec{n}_{k}^{\mathrm{in}}}$ stand for differentiations in the direction of the inner unit normal in $D_{k} \cup S, k=1,2$.

To prove the criterion for $V$ to be subharmonic on $\mathcal{O}$, it suffices to directly apply Lemma A. (After [21, Lemma 3.8.1], this lemma was reproved independently by Blanchet in [22, Theorem 3.1], see also [23, Lemma 4.1]). For that, we must put $D:=\mathcal{O}, D_{1}:=$ $\mathcal{O} \cap \mathbb{C}_{+}, D_{2}:=\mathcal{O} \cap \mathbb{C}_{-}, S:=\mathbb{R} \cap \mathcal{O}$ and rewrite (4.2) in the more explicit form (4.1).

We describe the Riesz measure of the function $V \in \operatorname{sbh}(\mathcal{O})$. For the role of the domain $D$, now we take an arbitrary disk $x_{0}+r \mathbb{D} \Subset \mathcal{O}$. We consider the open interval $\left(x_{0}-r, x_{0}+r\right) \Subset \mathcal{O} \cap \mathbb{R}$ and the upper and lower open half-disks

$$
D_{+}:=\left(x_{0}+r \mathbb{D}\right) \cap \mathbb{C}_{+}, \quad D_{-}:=\left(x_{0}+r \mathbb{D}\right) \cap \mathbb{C}_{-}
$$

in $\mathcal{O}$.

Applying the Green formula twice in each half-disk $D_{ \pm}$to a positive infinitely differentiable function $\psi \in\left(C_{0}^{\infty}(D)\right)^{+}$compactly supported in $D$ and to the function $V$ harmonic $D_{ \pm}$, and adding the results, we obtain

$$
\int_{D} V \Delta \psi=\int_{x_{0}-r}^{x_{0}+r} \psi(x)\left(\frac{\partial V}{\partial \vec{n}_{+}^{\text {in }}}+\frac{\partial V}{\partial \vec{n}_{-}^{\text {in }}}\right)(x) d x,
$$

where the right-hand side is positive by (4.1). It follows that after division by $2 \pi$, (4.1) gives the Riesz measure $\nu_{V}$ for the function $V$ subharmonic in $\mathcal{O}$.

If $V$ is positive and continuous on $\mathcal{O}_{0}$ and is subharmonic on the open set $\mathcal{O}_{0} \backslash A_{0}$, at every point $x_{0} \in Z_{0}$ we have the mean-value inequality

$$
0=V\left(x_{0}\right) \leq \frac{1}{2 \pi i} \int_{0}^{2 \pi} V\left(x_{0}+t e^{i \theta}\right) d \theta, \quad x_{0}+t \mathbb{D} \Subset \mathcal{O}_{0} .
$$

Consequently, $V$ is subharmonic in an open neighborhood $\mathcal{O} \cup\left(\mathcal{O}_{0} \backslash E_{0}\right)$ of the polar set $E_{0}$ and is locally upper bounded on $\mathcal{O} \cup \mathcal{O}_{0} \supset E_{0}$. By the theorem on removable singularities of subharmonic functions (see [24, Theorem 3.6.1]), the restriction of $V$ to 
$\mathcal{O} \cup \mathcal{O}_{0} \supset E_{0}$ admits a unique subharmonic extension to the entire set $\mathcal{O} \cup \mathcal{O}_{0}$; this extension is given by

$$
\limsup _{\substack{z \rightarrow x \\ z \in \mathcal{O}_{0} \backslash E_{0}}} V(z), \quad x \in E_{0} .
$$

Since $V$ is continuous on $\mathcal{O}_{0}$, the last upper limit coincides with $V(x), x \in E_{0}$, which completes the proof of Proposition 1 .

4.1. Extension of test functions to $\mathbb{C}_{*}$. Let $m \geq 2$. Given a test function $\varphi \in R \mathcal{P}_{0}^{m}$, we construct a function on $\mathbb{C}_{*}($ see $(2.9)-(2.10))$ :

$$
V^{\varphi}(\zeta):=\left(\mathrm{P}_{\mathbb{C}_{ \pm}} \varphi\right)(\zeta), \quad \zeta \in \mathbb{C}_{*} .
$$

Proposition 2. The function $V^{\varphi}$ is positive and subharmonic in the punctured plane $\mathbb{C}_{*}$, and its restriction $\left.V^{\varphi}\right|_{\mathbb{R}_{*}}$ coincides with $\varphi ;$ next, $V^{\varphi}$ satisfies the condition of seminormalization at zero:

$$
\limsup _{\substack{\zeta \rightarrow 0, \zeta \in \mathbb{C}_{*}}} \frac{V^{\varphi}(\zeta)}{-\log |\zeta|} \leq 1 .
$$

Furthermore, the constant $R_{\varphi}$ in (2.4) obeys the estimate

$$
V^{\varphi}(\zeta) \leq \text { const. }\left|\operatorname{Im} \frac{1}{\zeta}\right|, \quad|\zeta| \geq 2 R_{\varphi} .
$$

Moreover, we have

$$
\begin{aligned}
\left(\frac{\partial V^{\varphi}}{\partial \vec{n}_{+}^{\text {in }}}+\frac{\partial V^{\varphi}}{\partial \vec{n}_{-}^{\text {in }}}\right)(x) & \\
& :=\lim _{0<y \rightarrow 0} \frac{V^{\varphi}(x+i y)-V^{\varphi}(x)}{y}+\lim _{0>y \rightarrow 0} \frac{V^{\varphi}(x+i y)-V^{\varphi}(x)}{-y} \\
& =2 \lim _{0<y \rightarrow 0} \frac{V^{\varphi}(x+i y)-\varphi(x)}{y} \\
& =\frac{1}{\pi} \int_{\mathbb{R}_{*}} \frac{\varphi(t)-\varphi(x)}{(t-x)^{2}} d t=(-\mathrm{H} \varphi)^{\prime}(x) \geq 0, \quad x \in \mathbb{R}_{*} \backslash\left(E_{\varphi} \cup \partial_{\mathbb{R}} Z_{\varphi}\right),
\end{aligned}
$$

where $Z_{\varphi}$ and $E_{\varphi}$ are the sets occurring in (2.3E)-(2.3b), the left-hand side is the density for the restriction to $\mathbb{R}_{*} \backslash\left(E_{\varphi} \cup \partial_{\mathbb{R}} Z_{\varphi}\right)$ of the Riesz measure for the function $V^{\varphi} \in \operatorname{sbh}\left(\mathbb{C}_{*}\right)$.

Proof. Since $\varphi$ is positive, $V^{\varphi}$ is positive everywhere except zero. Next, $V^{\varphi}$ is continuous at the points of continuity of $\varphi$ and

$$
\lim _{\substack{\zeta \rightarrow x_{0} \\ \zeta \in \mathbb{C}_{*}}} V^{\varphi}(\zeta)=\varphi\left(x_{0}\right), \quad x_{0} \in \mathbb{R}_{*}
$$

by the well-known properties of the Poisson integral (see [25, Chapter I, Lemma 3.3]). Moreover, $V^{\varphi}$ is infinitely differentiable in $\mathbb{C}_{ \pm}$because it is harmonic in $\mathbb{C}_{ \pm}$. We need the following lemma, which is stated here only for the case of the plane $\mathbb{C}$.

Lemma B (see [26, Chapter I, §8, Theorem]). Suppose that a two times continuously differentiable curve $\Sigma$ is defined in a neighborhood of the point $z_{0} \in \mathbb{C}$. Suppose that, on one side of $\Sigma$, a harmonic function $V$ is defined in some neighborhood of $z_{0}$ (i.e., in a connected component of the set $\left(z_{0}+r \mathbb{D}\right) \cap(\mathbb{C} \backslash \Sigma)$ with $r$ sufficiently small $)$. Suppose also that for every $z \in \partial\left(\left(z_{0}+r \mathbb{D}\right) \cap \Sigma\right)$ the limit $\lim _{\zeta \rightarrow z} V(\zeta)=\psi(z)$ exists, and $\psi \in C^{2}(\Sigma)$. Then the gradient $\operatorname{grad} V$ has a finite limit at every point of the boundary $\partial\left(\left(z_{0}+r \mathbb{D}\right) \cap \Sigma\right)$ and can be extended by continuity to $\left(z_{0}+r \mathbb{D}\right) \cap \Sigma$. 
Let $x_{0} \in \mathbb{R}_{*} \backslash\left(E_{\varphi} \cup \partial_{\mathbb{R}} Z_{\varphi}\right)$, in the notation of formula (2.3) in Definition 1. We choose a number $r>0$ so small that

$$
\Sigma:=\left(x_{0}-r, x_{0}+r\right) \subset \mathbb{R}_{*} \backslash\left(E_{\varphi} \cup \partial_{\mathbb{R}} Z_{\varphi}\right) .
$$

By Lemma B, in the notation of (4.3), the corresponding restrictions of $V$ belong to the classes

$$
C^{1}\left(\bar{D}_{+} \backslash\left\{x_{0}-r, x_{0}+r\right\}\right), \quad C^{1}\left(\bar{D}_{+} \backslash\left\{x_{0}-r, x_{0}+r\right\}\right),
$$

where $\bar{D}_{+}$and $\bar{D}_{-}$are the closures of $D_{+}$and $D_{-}$in $\mathbb{C}$.

Since the point $x_{0} \in \mathbb{R}_{*} \backslash\left(E_{\varphi} \cup \partial_{\mathbb{R}} Z_{\varphi}\right)$ can be chosen arbitrarily, the function $V:=V^{\varphi}$ satisfies the conditions of Proposition 1 with $\mathcal{O}:=\mathbb{C}_{*} \backslash\left(E_{\varphi} \cup \partial_{\mathbb{R}} Z_{\varphi}\right)$. To prove that $V=V^{\varphi}$ is subharmonic in $\mathbb{C}_{*} \backslash\left(E_{\varphi} \cup \partial_{\mathbb{R}} Z_{\varphi}\right)$, it suffices to verify condition (4.1).

The function $V^{\varphi}(x+i y), \zeta=x+i y, x, y \in \mathbb{R}$, is odd in $y$ for every fixed $x$ by construction, see (4.4), (2.9)-(2.10). Consequently, for every $x \in \mathbb{R}_{*} \backslash\left(E_{\varphi} \cup \partial_{\mathbb{R}} Z_{\varphi}\right)$, the sum of the derivatives of $V$ in the directions of inner normals (i.e., the sum occurring in (4.1)) coincides in our case with

$$
\begin{aligned}
2 \lim _{\substack{y \rightarrow 0 \\
y>0}} & \frac{1}{y}\left(V^{\varphi}(x+i y)-\varphi(x)\right) \\
& =2 \lim _{\substack{y \rightarrow 0 \\
y>0}} \frac{1}{y}\left(\frac{1}{\pi} \int_{-\infty}^{+\infty} \frac{y}{(t-x)^{2}+y^{2}} \varphi(t) d t-\varphi(x)\right) \\
& =\frac{2}{\pi} \lim _{\substack{y \rightarrow 0 \\
y>0}} \int_{-\infty}^{+\infty} \frac{\varphi(t)-\varphi(x)}{(t-x)^{2}+y^{2}} d t=\frac{2}{\pi} f_{-\infty}^{+\infty} \frac{\varphi(t)-\varphi(x)}{(t-x)^{2}} d t .
\end{aligned}
$$

By the conjugate positivity condition (see (2.6)), the right-hand side in (4.10) is positive for every $x$. Consequently, condition (4.1) in Proposition 1 is fulfilled, and the function $V^{\varphi}$ is subharmonic in $\mathbb{C}_{*} \backslash\left(E_{\varphi} \cup \partial_{\mathbb{R}} Z_{\varphi}\right)$. Putting $A_{0}:=E_{\varphi} \cup \partial_{\mathbb{R}} Z_{\varphi}, E_{0}:=E_{\varphi}, Z_{0}:=\partial_{\mathbb{R}} Z_{\varphi}$ and applying the final part of Proposition 1, we see that $V^{\varphi}$ is subharmonic on the open set $\mathcal{O}_{0}:=\mathbb{C}_{*} \supset A_{0}$. Thus, $V^{\varphi} \in \operatorname{sbh}\left(\mathbb{C}_{*}\right)$. As a byproduct, identities (4.10) prove that (4.1) is fulfilled and, therefore, also (4.7), but without $(-\mathrm{H} \varphi)^{\prime}$ for the moment.

We pass to the verification of the seminormalization condition 3) in Definition 2, The seminormalization condition at zero (see (2.5)) implies that, for an arbitrary $\varepsilon>0$, for some $\alpha \in(0,1]$ depending only on $\varepsilon$ and $\varphi$ we have

$$
\varphi(x) \leq(1+\varepsilon) \log \frac{1}{|x|}, \quad|x| \leq \alpha .
$$

Moreover, by the compact support condition (2.4), in which we may assume that $R_{\varphi} \geq 1$, there exists a constant $C_{1} \geq 0$ such that

$$
\varphi(x) \leq C_{1}, \quad|x| \geq \alpha .
$$

Taking the finite support condition into account and coarsening (4.11) and (4.12) slightly, we may unite these two estimates:

$$
\varphi(x) \leq(1+\varepsilon) \log ^{+} \frac{e^{C_{1}} R_{\varphi}}{|x|}=:(1+\varepsilon) \log ^{+} \frac{C}{|x|}, \quad C:=e^{C_{1}} R_{\varphi}, \quad x \in \mathbb{R}_{*} .
$$

Since the Poisson kernel in (2.9) is positive, the last inequality implies

$$
V^{\varphi}(\zeta)=\left(\mathrm{P}_{\mathbb{C}_{ \pm}} \varphi\right)(\zeta) \leq(1+\varepsilon)\left(\mathrm{P}_{\mathbb{C}_{ \pm}} \log ^{+} \frac{C}{|\cdot|}\right)(\zeta), \quad \zeta \in \mathbb{C}_{*}
$$


It can easily be checked that for some constant $C_{2}$ depending only on $C$, we have

$$
\left(\mathrm{P}_{\mathbb{C}_{ \pm}} \log +\frac{C}{|\cdot|}\right)(\zeta) \leq \log \frac{1}{|\zeta|}+C_{2}, \quad \zeta \in \mathbb{D}_{*} .
$$

By (4.14), it follows that

$$
V^{\varphi}(\zeta):=\left(\mathrm{P}_{\mathbb{C}_{ \pm}} \varphi\right)(\zeta) \leq \log \frac{1}{|\zeta|}+C_{2}, \quad \zeta \in \mathbb{D}_{*}
$$

Therefore,

$$
\limsup _{\substack{\zeta \rightarrow 0 \\ \zeta \in \mathbb{C}_{*}}} \frac{V^{\varphi}(\zeta)}{-\log |\zeta|} \leq 1+\varepsilon
$$

Since $\varepsilon>0$ is arbitrary, we obtain the seminormalization condition (4.5).

We pass to the proof of (4.6). For $\zeta=x+i y,|\zeta|=r \geq 2 R_{\varphi}$, we consider separately the case where $|x| \geq y$ and $y>|x|$. It suffices to give a proof for the closed upper half-plane, that is, for $y \geq 0$. If $|x| \geq y$, then for $|t| \leq R_{\varphi}$ we have

$$
(t-x)^{2} \geq(|x|-|t|)^{2} \geq\left(r / \sqrt{2}-R_{\varphi}\right)^{2} \geq(r / \sqrt{2}-r / 2)^{2}=\left(\frac{\sqrt{2}-1}{2}\right)^{2} r^{2} .
$$

Consequently, if $|x| \geq y$ and $r \geq 2 R_{\varphi}$, then

$$
V^{\varphi}(\zeta) \leq \frac{1}{\pi} \int_{-R_{\varphi}}^{R_{\varphi}} \varphi(t) \frac{y}{(t-x)^{2}} d t \leq \text { const. } \int_{-R_{\varphi}}^{R_{\varphi}} \varphi(t) d t \cdot \frac{y}{r^{2}} \leq \text { const. }\left|\operatorname{Im} \frac{1}{\zeta}\right| .
$$

Similarly, for $|x|<y$ and $r \geq 2 R_{\varphi}$ we obtain

$$
V^{\varphi}(\zeta) \leq \frac{1}{\pi} \int_{-R_{\varphi}}^{R_{\varphi}} \varphi(t) \frac{y}{y^{2}} d t \leq \text { const. } \int_{-R_{\varphi}}^{R_{\varphi}} \varphi(t) d t \cdot \frac{2 y}{r^{2}} \leq \text { const. }\left|\operatorname{Im} \frac{1}{\zeta}\right| .
$$

So, estimate (4.6) is proved.

Finally, to verify the last identity in (4.7), we act as in [27, Chapter XI, $\S \mathrm{E}, 2$, Remark]. Consider the following two functions:

$$
\begin{aligned}
& \left(\mathrm{P}_{\mathbb{C}_{ \pm}} \varphi\right)(z), \quad(H \varphi)(z):=\frac{1}{\pi} \int_{-\infty}^{\infty}\left(\frac{\operatorname{Re} z-t}{|z-t|^{2}}+\frac{t}{t^{2}+1}\right) \varphi(t) d t \\
& z \in \mathbb{C}_{+} \cdot\left(\frac{\operatorname{Re} z-t}{|z-t|^{2}}+\frac{t}{t^{2}+1}\right) \varphi(t) d t, \quad z \in \mathbb{C}_{+} .
\end{aligned}
$$

They are harmonic in $\mathbb{C}_{+}$and have continuous second derivatives, maybe, outside an exceptional set $E_{\varphi} \cup \partial_{\mathbb{R}} Z_{\varphi}$. By the Cauchy-Riemann equations, we obtain

$$
\begin{aligned}
(H \varphi)^{\prime}(x) & =\lim _{y \rightarrow 0+} \frac{\partial(H \varphi)(x+i y)}{\partial x} \\
& =-\lim _{x \rightarrow 0+} \frac{\partial\left(\mathrm{P}_{\mathbb{C}_{ \pm}} \varphi\right)(x+i y)}{\partial y}=\lim _{y \rightarrow 0+} \frac{\varphi(x)-\left(\mathrm{P}_{\mathbb{C}_{ \pm}} \varphi\right)(x+i y)}{y},
\end{aligned}
$$

where the last limit can be written in the form

$$
\frac{1}{\pi} \int_{0}^{+\infty} \frac{2 \varphi(x)-\varphi(x+t)-\varphi(x-t)}{t^{2}} d t .
$$

Together with the fact (mentioned in the Introduction) that conditions (2.6) and (2.7) coincide, this gives the identity in (2.6) and the last identity in (4.7). 
4.2. Restriction of Jensen potentials to $\mathbb{R}_{*}:=\mathbb{R} \backslash\{0\}$. The following statement is a sort of converse to Proposition 2

Proposition 3. If $V$ is a Jensen potential continuous on $\mathbb{R}_{*}$, and its restriction $\varphi:=$ $\left.V\right|_{\mathbb{R}_{*}}$ belongs to $C^{m}\left(\mathbb{R}_{*} \backslash\left(Z_{V} \cup E_{V}\right)\right)$ for some $m \in(\mathbb{N} \backslash\{1\}) \cup\{\infty\}$, where $Z_{V}:=\{x \in$ $\left.\mathbb{R}_{*}: V(x)=0\right\}$ and $E_{V} \subset \mathbb{R}_{*}$ is a closed polar subset of $\mathbb{C}$, then $\varphi \in R \mathcal{P}_{0}^{m}$ and

$$
V^{\varphi}(\zeta):=\left(\mathrm{P}_{\mathbb{C}_{ \pm}} \varphi\right)(\zeta) \geq V(\zeta), \quad \zeta \neq 0
$$

Proof. The conditions of positivity, compact support (2.4), and seminormalization at zero (2.5) (see the definition of the classes $R \mathcal{P}_{0}^{m}$ ) are fulfilled by the Definition 2 of the Jensen potentials. The function $V^{\varphi}$ is continuous on $\mathbb{C}_{*}$ (see [25, Chapter I, Lemma 3.3]) and is harmonic in $\mathbb{C}_{ \pm}$. Since the Jensen potential $V$ is subharmonic in $\mathbb{C}_{*}$ and its restriction to $\mathbb{R}_{*}$ coincides with the boundary values of the function $V^{\varphi}$ on $\mathbb{R}_{*}$, it follows that $V^{\varphi}$ majorizes $V$ on $\mathbb{C}_{*}$, i.e., we have (4.15). For every $x_{0} \in \mathbb{R}_{*}$, the mean value of $V^{\varphi}$ on the disk $x_{0}+r \mathbb{D}, r<\left|x_{0}\right|$, does not exceed the mean value of $V$ on the same disk. Since $V$ is subharmonic, the latter quantity is greater than or equal to $\varphi\left(x_{0}\right)=V^{\varphi}\left(x_{0}\right)$. Therefore, $V^{\varphi}$ is subharmonic in $\mathbb{C}_{*}$. Now, the requirement that the inverse Hilbert transform $-H \varphi$ should be monotone increasing on each connected component of the set $\mathbb{R}_{*} \backslash\left(Z_{\varphi} \cup E_{\varphi}\right)$ (open in $\mathbb{R}_{*}$ ) can be verified in the form of the conjugate positivity condition (2.6). For this, we use the theory of distributions.

Let $x_{0} \in \mathbb{R}_{*} \backslash\left(E_{\varphi} \cup Z_{\varphi}\right)$. Choose $r>0$ so small that

$$
\Sigma:=\left(x_{0}-r, x_{0}+r\right) \subset \mathbb{R}_{*} \backslash\left(E_{\varphi} \cup Z_{\varphi}\right) .
$$

By Lemma B, in the notation (4.3), the corresponding restrictions of $V^{\varphi}$ belong to the classes (4.9), and even they are infinitely differentiable on $D_{+}$and $D_{-}$, because they are harmonic.

Let $\psi_{0} \in C_{0}^{\infty}\left(x_{0}-r, x_{0}+r\right):=C_{0}^{\infty}\left(\left(x_{0}-r, x_{0}+r\right)\right)$ be a positive function. We write the second Green formula in these half-disks for a positive extension $\psi \in C_{0}^{\infty}\left(x_{0}+r \mathbb{D}\right)$ of $\psi_{0}=\left.\psi\right|_{\left(x_{0}-r, x_{0}+r\right)}$, and for $V^{\varphi}$ :

$$
\begin{aligned}
& \text { (4.17+) } \int_{x_{0}-r}^{x_{0}+r}\left(V^{\varphi}(t) \frac{\partial \psi}{\partial \vec{n}_{+}^{\text {in }}}(t)-\psi(t) \frac{\partial V^{\varphi}}{\partial \vec{n}_{+}^{\text {in }}}(t)\right) d t=\iint_{D_{+}}\left(\psi \Delta V^{\varphi}-V^{\varphi} \Delta \psi\right) d x d y \\
& \text { (4.17-) } \int_{x_{0}-r}^{x_{0}+r}\left(V^{\varphi}(t) \frac{\partial \psi}{\partial \vec{n}_{-}^{\text {in }}}(t)-\psi(t) \frac{\partial V^{\varphi}}{\partial \vec{n}_{-}^{\text {in }}}(t)\right) d t=\iint_{D_{-}}\left(\psi \Delta V^{\varphi}-V^{\varphi} \Delta \psi\right) d x d y \text {. }
\end{aligned}
$$

Since $\psi$ is smooth and the normals $\vec{n}_{+}^{\text {in }}$ and $\vec{n}_{-}^{\text {in }}$ are positively oriented, we have

$$
\frac{\partial \psi}{\partial \vec{n}_{+}^{\text {in }}}(t)+\frac{\partial \psi}{\partial \vec{n}_{-}^{\text {in }}}(t)=\frac{\partial \psi}{\partial \vec{n}_{+}^{\text {in }}}(t)+\frac{\partial \psi}{\partial\left(-\vec{n}_{+}^{\text {in }}\right)}(t) \equiv 0, \quad t \in\left(x_{0}+r, x_{0}+r\right) .
$$

Since $V^{\varphi}$ is harmonic in $\mathbb{C}_{ \pm}$, we obtain $\Delta V^{\varphi}(\zeta) \equiv 0$ on $D_{+} \cup D_{-}$. Together with (4.18) and after adding (4.17\#) and (4.17円), the last identity yields

$$
\int_{x_{0}-r}^{x_{0}+r} \psi_{0}(t)\left(\frac{\partial V^{\varphi}}{\partial \vec{n}_{+}^{\text {in }}}(t)+\frac{\partial V^{\varphi}}{\partial \vec{n}_{-}^{\text {in }}}(t)\right) d t=\iint_{x_{0}+r \mathbb{D}} V^{\varphi} \Delta \psi d x d y,
$$

where the right-hand side is positive because $V^{\varphi}$ is subharmonic in $x_{0}+r \mathbb{D}$ (from a distributional point of view). Thus, formula (4.19) implies

$$
\frac{\partial V^{\varphi}}{\partial \vec{n}_{+}^{\text {in }}}(t)+\frac{\partial V^{\varphi}}{\partial \vec{n}_{-}^{\text {in }}}(t) \geq 0, \quad t \in \mathbb{R}_{*} \backslash\left(E_{\varphi} \cup Z_{\varphi}\right),
$$

because $x_{0} \in \mathbb{R}_{*}, r$ in (4.16), and a positive function $\psi_{0} \in C_{0}^{\infty}\left(x_{0}-r, x_{0}+r\right)$ can be taken arbitrarily. But the sum of the normal derivatives in (4.20) was already calculated 
in (4.10), and it is equal to the Cauchy principal value of the integral in (2.6). Thus, the conjugate positivity condition (2.6) holds true, and $\varphi$ belongs to the test class $R \mathcal{P}_{0}^{m}$.

Examples. Proposition 3 provides the simplest examples of test functions in the class $R \mathcal{P}_{0}^{\infty}$, obtained by restriction to $\mathbb{R}_{*}$ of known Jensen potentials or Green functions for domains $D \ni 0$ with pole at zero, because such functions are a particular case of Jensen potentials. Here are some of them, taken from [18, Example 1] (as usual, $f^{+}=\max \{0, f\}$ ): 1) $\varphi(t):=\log ^{+} \frac{R}{|t|}, t \in \mathbb{R}_{*}$, the restriction of a Jensen potential, namely, of the extended Green function for the disk $R \mathbb{D}$ with pole at zero: $z \mapsto \log ^{+} \frac{R}{|z|}, z \in \mathbb{C}_{*}$ (see [1, $\S 4$, Example 1]);

2) $\varphi(t)=\log ^{+} \frac{R}{|t|}-\frac{1}{2}\left(1-\frac{t^{2}}{R^{2}}\right)^{+}, t \in \mathbb{R}_{*}$ (see [1, §4, Example 1]);

3 ) the restriction to $\mathbb{R}^{*}$ of the positive Green function with pole at zero for a bounded domain $D$ in $\mathbb{C}$ with $C^{1}$-boundary and with $0 \in D$ (see [1, §4, Example 3]).

Explicit test functions of more complicated structure that belong to $R \mathcal{P}_{0}^{\infty}$ will emerge in the sequel in the uniqueness theorems from $₫ 6$.

\section{$\S 5$. Proofs of the MAIN THEOREMS}

5.1. Proof of Theorem 1 on the uniqueness sequences for a Bernstein space. 1. Proof of the implications 1) $\Longrightarrow 2$ ), 3). Suppose that the quantities 2) in (2.11) or 3) in Theorem 1 are finite. Then the functions $\varphi \in R \mathcal{P}_{0}^{m}$ that, in the form $\left.\varphi\right|_{\mathbb{R}_{*}}$, are associated with arbitrary Jensen potentials $V$ (see Proposition 3 ) with the estimate (4.15) are such that the quantity (3.5) in Theorem 3 (occurring in 2)) is finite, because (4.15) holds true for every $\lambda_{k}$, and $\varphi$ and $V$ coincide on the real axis by construction. By Theorem 3 , the finiteness of the lower upper bound (3.5) implies that $\Lambda$ is a nonuniqueness sequence for the Bernstein space $B_{\sigma}^{\infty}$. This proves the implications 1) $\Longrightarrow 2$ ) and 1) $\Longrightarrow 3$ ) of Theorem 1 .

2. Proof of the implications 2),3) $\Longrightarrow$ 1) in Theorem 1. Suppose now that $\Lambda$ is a nonuniqueness sequence for the Bernstein space $B_{\sigma}^{\infty}$. Then, by the equivalence 1) $\Longleftrightarrow 3$ ) in Theorem 3 there exists a constant $C$ such that for all Jensen potentials $V \in \mathcal{P}_{J_{0}}$ we have

$$
\sum V\left(\lambda_{k}\right) \leq \frac{\sigma}{\pi} \int_{-\infty}^{+\infty} V(t) d t+C
$$

where the constant $C$ is independent of $V \in \mathcal{P}_{J_{0}}$.

Now, we choose an arbitrary test function $\varphi \in R \mathcal{P}_{0}^{m}$ and, as in (4.4), consider the Poisson transform

$$
V^{\varphi}(\zeta):=\left(\mathrm{P}_{\mathbb{C}_{ \pm}} \varphi\right)(\zeta), \quad \zeta \in \mathbb{C}_{*}
$$

By Proposition 2, this function $V^{\varphi}$ possesses nearly all properties of a Jensen potential. Specifically, it is subharmonic and positive outside zero, is seminormalized at zero (see (4.5)), and obeys the conjugate positivity condition (2.6)-(2.7). The only possible exception is the compact support condition (see (4.6)), which is replaced by the weaker condition (4.6):

$$
V^{\varphi}(\zeta) \leq b\left|\operatorname{Im} \frac{1}{\zeta}\right|, \quad|\zeta| \geq r, b, r \geq 0 \text { are constants. }
$$

It is quite easy to convert such a function into a Jensen potential: it suffices to consider the function

$$
V_{\varepsilon}^{\varphi}(\zeta):=\left(V^{\varphi}(\zeta)-\varepsilon\right)^{+}
$$


with arbitrary $\varepsilon>0$, which already has compact support but has also all the previous properties (that is, this is a true Jensen potential continuous outside zero). As was mentioned in (5.1), such potentials satisfy the inequalities

$$
\sum_{k} V_{\varepsilon}^{\varphi}\left(\lambda_{k}\right) \leq \frac{\sigma}{\pi} \int_{-\infty}^{+\infty} V_{\varepsilon}^{\varphi}(t) d t+C=\frac{\sigma}{\pi} \int_{-\infty}^{+\infty}(\varphi(t)-\varepsilon)^{+} d t+C,
$$

where the constant $C$ does not depend on a Jensen potential $V_{\varepsilon}^{\varphi} \in \mathcal{P}_{J_{0}}$ (in particular, on the number $\varepsilon$ ). Letting $\varepsilon>0$ tend to zero and using (5.2), we obtain

$$
\sum_{k}\left(\mathrm{P}_{\mathbb{C}_{ \pm}} \varphi\right)\left(\lambda_{k}\right) \leq \frac{\sigma}{\pi} \int_{-\infty}^{+\infty} \varphi(t) d t+C,
$$

and the finiteness of the suprema in 2), (2.11), and 3) follows. This proves the implications $2), 3) \Longrightarrow 1$ ) in Theorem 1 .

\subsection{Proof of Theorem 2 on the completeness of systems of exponentials in spaces on an interval.}

1. Conditions of completeness. The sequences of exponents for collections of exponentials complete in a function space on an interval are in a well-known relationship with uniqueness sequences in the dual space realized as a special space of entire functions of exponential type (see [2, [18, Theorem 2.1.1]). This relationship shows that if $\Lambda$ is a uniqueness sequence for $B_{\sigma}^{\infty}$, then the system is complete in any space among $C_{\mathbb{C}}\left(\bar{I}_{2 \sigma}\right)$ and $L^{p}\left(I_{2 \sigma}\right), p \geq 1$, because the duals to these spaces, when represented as spaces of entire functions, are all included in the Bernstein space $B_{\sigma}^{\infty}$.

2. Noncompleteness and estimates for the completeness excess. Suppose now that the suprema in (2.11) or in (2.12) (under the restriction $\Lambda \subset \mathbb{R}$ ) are finite. Then by Theorem 1, $\Lambda$ is a nonuniqueness sequence for $B_{\sigma}^{\infty}$. At this point, a case-by-case analysis is required.

The case of the spaces $C_{\mathbb{R}}\left(\bar{I}_{2 \sigma}\right)$ and $L^{p}\left(I_{2 \sigma}\right)$ for $p \geq 2$. Conversely, let now the suprema in (2.11) or (under the assumption $\Lambda \subset \mathbb{R}$ ) in (2.12) be bounded above. Then $\Lambda$ is a nonuniqueness sequence for $B_{\sigma}^{\infty}$, and there exists a nonzero entire function $f$ satisfying (1.4), bounded on the real axis, and vanishing on $\Lambda$. There is no loss of generality in assuming that $\Lambda$ is nonempty and there exists a point $\lambda^{\prime} \in \Lambda$.

Then the nonzero entire function $F(z):=f(z) /\left(z-\lambda^{\prime}\right)$ of exponential type at most $\sigma$ vanishes on the sequence $\Lambda^{\prime}:=\Lambda \backslash \lambda^{\prime}$ and, at te same time, belongs to $L_{\mathbb{C}}^{2}(\mathbb{R})$. By the classical Paley-Wiener theorem, it follows that there exists a function $g \in L^{2}(-\sigma, \sigma)$ such that

$$
F(z)=\int_{-\sigma}^{\sigma} e^{i z t} g(t) d t, \quad F\left(\Lambda^{\prime}\right)=0
$$

By the well-known duality (see [18, Theorem 2.1.1]), this means that the system $\operatorname{Exp}^{\Lambda^{\prime}}$ is not complete in $L^{2}(-\sigma, \sigma)$. But the condition $g \in L^{2}(-\sigma, \sigma)$ (for $\sigma<\infty$ ) shows that, for every $1 \leq q<2$, we still have $g \in L^{q}(-\sigma, \sigma)$ and, moreover, $g$ is a density of a Borel measure. By the same duality, the system $\operatorname{Exp}^{\Lambda^{\prime}}$ is not complete in any space among $L^{p}\left(I_{2 \sigma}\right)$ for $p \geq 2,1 / p+1 / q=1$, and $C_{\mathbb{R}}\left(\bar{I}_{2 \sigma}\right)$. The removal of one exponent to attain noncompleteness means precisely (see [18, 1.1.3]) that exc $\Lambda \leq 0$.

The case of the spaces $L^{p}\left(I_{2 \sigma}\right)$ for $1 \leq p<2$. If a sequence $\Lambda \subset \mathbb{C}, 0 \notin \Lambda$, does not obey condition (2.11) or, under the restriction $\Lambda \subset \mathbb{R}$, it does not obey condition (2.12), then by Theorem 1, $\Lambda$ is still a nonuniqueness sequence for $B_{\sigma}^{\infty}$, and there exists a nonzero entire function $f$ that satisfies (1.4), is bounded on the real axis, and vanishes on $\Lambda$. There is no loss of generality in assuming that $\Lambda$ contains at least two (possibly, 
multiple) points $\lambda^{\prime}, \lambda^{\prime \prime} \in \Lambda$, because a system of two exponentials is not complete an any of the spaces in question. Now, consider the function

$$
F(z):=\frac{f(z)}{\left(z-\lambda^{\prime}\right)\left(z-\lambda^{\prime \prime}\right)}
$$

which, clearly, is a nonzero entire function of exponential type at most $\sigma$, and it vanishes on $\Lambda^{\prime \prime}:=\Lambda \backslash\left\{\lambda^{\prime}, \lambda^{\prime \prime}\right\}$. Furthermore, $F \in L^{p}(\mathbb{R}), 1 \leq p \leq 2$, and by the Hausdorff-Young theorem there exists a function $g \in L^{q}(-\sigma, \sigma), 1 / p+1 / q=1$ (see the generalization of the Paley-Wiener Theorem in [7, 1.7] or [8, 1.7]) such that (compare with (5.4)) we have

$$
F(z)=\int_{-\sigma}^{\sigma} e^{i z t} g(t) d t, \quad F\left(\Lambda^{\prime \prime}\right)=0 .
$$

By the well-known duality [18, Theorem 2.1.1], this means that the system $\operatorname{Exp}^{\Lambda^{\prime \prime}}$ is not complete in $L^{p}(-\sigma, \sigma)$, where $p \leq 2$. Removal of two exponentials for attaining noncompleteness means precisely (see [18, 1.1.3]) that exc $\Lambda \leq 1$.

Note that, under conditions (2.11) or (2.12), the test classes $R \mathcal{P}_{0}^{m}$ can always be replaced with the narrower classes of item 3) in Theorem 1.

\section{$\S 6$. Some initial applications}

Our Theorems 1 and 2 imply a number of results about (non)uniqueness sequences for Bernstein spaces and about the completeness of systems of exponentials in $C\left(\bar{I}_{d}\right)$ and $L^{p}\left(I_{d}\right)$ (for instance, the celebrated Beurling-Malliavin theorem on the radius of completeness stated in Subsection 1.2, but the new proof of it is neither shorter, nor simpler than an earlier proof presented in [28, see also the exposition in [29, Chapter III]). So, here we restrict ourselves to simplest consequences of Theorems 1 and 2 which illustrate the specific features of the new approach. Proofs of many other known results from the monographs [5, 1, 7, 8, 9, their generalizations, and, as far as possible, new facts will be exposed elsewhere.

6.1. Uniqueness theorems. It is most easy to apply Theorems 1 and 2 for obtaining conditions sufficient for uniqueness in Bernstein spaces and conditions for completeness of systems of exponentials in $C\left(\bar{I}_{d}\right)$ and $L^{p}\left(I_{d}\right)$. First, we note that in the proofs of Theorems 1 and 2, as well as in their predecessors Theorem A and Theorem 3 (see [1]), no conditions were imposed except the absence of a limit point for the sequence in question (or the sequence of exponents) and the nonessential requirement that the sequence should not contain zero (see Remark 2). Below we show that all sufficient conditions for uniqueness from the Cartwright theorem (see Subsection 1.2) and the commentaries after it are contained in Theorem 1. In fact, all of them are covered by the following simple consequence of Theorems 1 and 2.

Corollary 1. Let $\varphi \in R \mathcal{P}_{0}^{m}$ be a nonzero function, and let $\sigma>0$. If for a sequence (1.1) we have

$$
\limsup _{r \rightarrow+\infty}\left(\sum_{k}\left(\mathrm{P}_{\mathbb{C}_{ \pm}} \varphi\right)\left(\frac{\lambda_{k}}{r}\right)-r \frac{\sigma}{\pi} \int_{\mathbb{R}} \varphi(x) d x\right)=+\infty,
$$

then $\Lambda$ is a uniqueness sequence for $B_{\sigma}^{\infty}$, and the system of exponentials $\operatorname{Exp}^{\Lambda}$ is complete in any of the spaces $C\left(\bar{I}_{2 \sigma}\right)$ and $L^{p}\left(I_{2 \sigma}\right), p \geq 1$.

The proof of this corollary readily follows from Theorems 1 and 2 if we use the invariance of the class $R \mathcal{P}_{0}^{m}$ under homothety, as was mentioned after Definition 1 in (2.8), and make the change of variables $x=t / r$ in the Poisson integrals and the last integral. 
Examples. We apply Corollary 1 to certain specific functions $\varphi$ in the class $R \mathcal{P}_{0}^{m}$.

1. Let $\varphi$ be the function in Example 1) in Subsection 4.2 with $R=1$, i.e., $\varphi(t)=$ $\log ^{+} \frac{1}{|t|}$. Then condition (6.1) for being a uniqueness sequence can be written in the form

$$
\limsup _{r \rightarrow+\infty}\left(\sum_{\operatorname{Im} \lambda_{k} \neq 0} \frac{1}{\pi} \int_{-1}^{1}\left|\operatorname{Im} \frac{1}{x-\lambda_{k} / r}\right| \log \frac{1}{|x|} d x+\sum_{\operatorname{Im} \lambda_{k}=0} \log ^{+} \frac{\left|\lambda_{k}\right|}{r}-\frac{2 \sigma}{\pi} r\right)=+\infty \text {. }
$$

In particular, the first sum here is majorized by

$$
\sum_{\operatorname{Im} \lambda_{k} \neq 0} \log +\frac{\left|\lambda_{k}\right|}{r}
$$

(see Proposition 3, inequality (4.15)). Thus, (6.2) implies also a weaker condition that guarantees uniqueness, namely,

$$
\limsup _{r \rightarrow+\infty}\left(\sum_{k} \log ^{+} \frac{\left|\lambda_{k}\right|}{r}-\frac{2 \sigma}{\pi} r\right) \stackrel{(1.8)}{=} \limsup _{r \rightarrow+\infty}\left(N_{\Lambda}(r)-\frac{2 \sigma}{\pi} r\right)=+\infty,
$$

which is in full accordance with the Levinson theorem (see Subsection 1.2) with $p=+\infty$, but, surely, does not prove it completely for $p<+\infty$. In particular, this implies some elementary facts: if $\Lambda$ is a nonuniqueness sequence for $B_{\sigma}^{\infty}$, or $\operatorname{Exp}^{\Lambda}$ is not complete in $C\left(\bar{I}_{d}\right)$ or $L^{p}\left(I_{d}\right)$, then $\Lambda$ must have finite averaged upper density; consequently, it must be of finite upper density because $n_{\Lambda}(t) \leq N_{\Lambda}$ (et) for all $t>0$, the convergence exponent does not exceed 1 and, a fortiori, the series (1.6) converges.

At the same time, condition (6.3) is indeed weaker than (6.2), because now we can deduce the Cartwright theorem (see Subsection 1.2) from it. Suppose that $\Lambda$ is a nonuniqueness sequence for some $B_{\sigma}^{\infty}$. Then the first sum in (6.2) is finite, for example, for $r=1$. Summation and integration can be interchanged in this case, because all summands and the function $\varphi$ are positive, see [16, Chapter IV, $\S 4$, Corollary 4]. Therefore, the series (1.5) converges for at least one $t \in(-1,1)$, the convergence of the series (1.6) implies that of the series (1.5) for every $t$, and the Cartwright theorem follows.

2. Let $\varphi$ be the function in Example 2) in Subsection 4.2 with $R=1$, i.e.,

$$
\varphi(t)=\log ^{+} \frac{1}{|t|}-\frac{1}{2}\left(1-t^{2}\right)^{+}, \quad t \in \mathbb{R}_{*} .
$$

Then condition (6.1) for uniqueness sequences can be rewritten in the form

$$
\begin{aligned}
\limsup _{r \rightarrow+\infty}\left(\sum_{\operatorname{Im} \lambda_{k} \neq 0} \frac{1}{\pi} \int_{-1}^{1}\left|\operatorname{Im} \frac{1}{x-\lambda_{k} / r}\right|\left(\log \frac{1}{|x|}-\frac{1}{2}\left(1-x^{2}\right)\right) d x\right. \\
\left.+\sum_{\operatorname{Im} \lambda_{k}=0}\left(\log ^{+} \frac{r}{\left|\lambda_{k}\right|}-\frac{1}{2}\left(1-\frac{\left|\lambda_{k}\right|^{2}}{r^{2}}\right)^{+}\right)-\frac{4 \sigma}{3 \pi} r\right)=+\infty
\end{aligned}
$$

In particular, the first sum here is at least

$$
\sum_{\operatorname{Im} \lambda_{k} \neq 0}\left(\log ^{+} \frac{r}{\left|\lambda_{k}\right|}-\frac{1}{2}\left(1-\frac{\left|\lambda_{k}\right|^{2}}{r^{2}}\right)^{+}\right)
$$

(see Proposition 3, inequality (4.15) ). Thus, formula (6.4) implies the following condition for being a uniqueness sequence:

$$
\limsup _{r \rightarrow+\infty}\left(\sum_{\left|\lambda_{k}\right| \leq r}\left(\log \frac{r}{\left|\lambda_{k}\right|}-\frac{1}{2}\left(1-\frac{\left|\lambda_{k}\right|^{2}}{r^{2}}\right)\right)-\frac{4 \sigma}{3 \pi} r\right)=+\infty .
$$


Representing the sum here as a Stiltjes integral, after a series of integrations by parts we see that condition (6.5) for nonuniqueness sequences coincides with the relation

$$
\limsup _{r \rightarrow+\infty}\left(\frac{1}{r^{2}} \int_{0}^{r} N_{\Lambda}(t) t d t-\frac{4 \sigma}{3 \pi} r\right)=+\infty .
$$

It can easily be shown that if the last condition is fulfilled, then (6.3) holds true, i.e., we obtain nothing new in (6.6) compared to (6.3). However, we do not know if the same happens with condition (6.4).

3. We show an "asymmetric" example of application of Corollary 1 .

Let $\zeta=t e^{i \theta} \in \mathbb{C}$, and let $k$ be a $\gamma$-trigonometrically convex (se 6 [2]) continuous $2 \pi$-periodic positive function. Throughout this item, we assume that $\max k \leq 1$. For $a>1$ and $t>1$, we put

$$
V_{k}(\zeta ; a)=\frac{a^{\gamma}}{2 \gamma\left(a^{2 \gamma}+1\right)} k(\theta)\left(\left(\frac{a}{t}\right)^{\gamma}-\left(\frac{t}{a}\right)^{\gamma}\right)^{+}
$$

and for $0<t<1$ we put

$$
V_{k}(\zeta ; a)=\frac{a^{\gamma}}{2 \gamma\left(a^{2 \gamma}+1\right)} k(\theta)\left((a t)^{\gamma}-\left(\frac{1}{a t}\right)^{\gamma}\right)^{+}+\log ^{+} \frac{1}{t} .
$$

In $[18, \S 7]$ it was shown that the functions defined above are continuous Jensen potentials smooth outside the points $t=a$ and $t=1$. Accordingly, by Proposition 3 , their restrictions to $\mathbb{R}_{*}$ belong to the class $R \mathcal{P}_{0}^{\infty}$ of test functions. Here we restrict ourselves to the simplest example of the function

$$
k(\theta)=\frac{1}{2}|1+\cos \theta|, \quad V_{k}:=V_{k}(\cdot ; a) .
$$

In this case it is easy to check that the function (6.9) is $\gamma$-trigonometrically convex for every $\gamma \geq 1 / 2$. The replacement of the Poisson integrals in items $2-3$ of Theorem 1 at the points $\lambda_{k}$ by the Jensen potentials themselves will only lead to smaller quantities (see Proposition 3, inequality 4.15). So, in the case under study, in Corollary 1 we can choose the functions (6.7)- (6.8) themselves in place of the Poisson integrals.

Let $\gamma \geq 1 / 2$. For simplicity, suppose that $\gamma<1$. If the function $k$ is chosen as in (6.9), the function $V$ is chosen as in (6.7)-(6.8), and

$$
\begin{aligned}
\limsup _{r \rightarrow+\infty}\left(\sum_{k} V\left(\lambda_{k} / r ; a\right)-r \frac{\sigma}{\pi}((a-1 / a)\right. & \frac{2 \gamma}{1-\gamma^{2}}-\frac{1}{1-\gamma}\left(a^{\gamma}-a^{-\gamma}\right) \\
& \left.\left.+\frac{1}{1+\gamma}\left(a^{-\gamma}-a\right)+\frac{2 \gamma\left(a^{2 \gamma}+1\right)}{a^{\gamma}}\right)\right)=+\infty,
\end{aligned}
$$

then $\Lambda$ is a uniqueness sequence for $B_{\sigma}^{\infty}$.

The cases where $\gamma=1$ and $\gamma>1$ are much subtler, and we do not consider them here (cf. [18, §7, Uniqueness theorem, items 2)-3)]).

We present a short proof of the Schwartz theorem stated in the Introduction.

Proof of the Schwartz theorem. Under condition (1.9), there is no loss of generality in assuming that for sufficiently small $\varepsilon>0$ we have

$$
\sum_{k \in \mathbb{N}}\left|\operatorname{Im} \frac{1}{\lambda_{k}}\right|<\varepsilon
$$

in place of the second condition in (1.9). To ensure this, it suffices to shift a finite number of points in $\Lambda$; this does not affect (non)completeness, see Remark 2 , The first estimate

\footnotetext{
${ }^{6}$ The main condition is that $k^{\prime \prime}+\gamma^{2} k \geq 0$ in the sense of the theory of distributions.
} 
in (1.9) implies $\left|\operatorname{Im} \lambda_{k}\right| \geq\left|\lambda_{k}\right| \sin \alpha$. This yields an estimate for the Poisson kernel at every point $\lambda_{k}$ :

$$
\frac{1}{\pi} \frac{\left|\operatorname{Im} \lambda_{k}\right|}{\left(t-\operatorname{Re} \lambda_{k}\right)^{2}+\left(\operatorname{Im} \lambda_{k}\right)^{2}} \leq \frac{1}{\pi \sin ^{2} \alpha} \frac{\left|\operatorname{Im} \lambda_{k}\right|}{\left|\lambda_{k}\right|^{2}}=\frac{1}{\pi \sin ^{2} \alpha}\left|\operatorname{Im} \frac{1}{\lambda_{k}}\right| .
$$

By (6.10), it follows that for every test function $\varphi \in R \mathcal{P}_{0}^{m}$ we have

$$
\begin{aligned}
\sum_{k \in \mathbb{N}}\left(\mathrm{P}_{\mathbb{C}_{ \pm}} \varphi\right)\left(\lambda_{k}\right) & :=\sum_{k \in \mathbb{N}} \frac{1}{\pi} \int_{-\infty}^{+\infty} \frac{\left|\operatorname{Im} \lambda_{k}\right|}{\left(t-\operatorname{Re} \lambda_{k}\right)^{2}+\left(\operatorname{Im} \lambda_{k}\right)^{2}} \varphi(t) d t \\
& \leq \sum_{k \in \mathbb{N}} \frac{1}{\pi \sin ^{2} \alpha}\left|\operatorname{Im} \frac{1}{\lambda_{k}}\right| \cdot \int_{-\infty}^{+\infty} \varphi(t) d t \leq \frac{\varepsilon}{\pi \sin ^{2} \alpha} \cdot \int_{-\infty}^{+\infty} \varphi(x) d x .
\end{aligned}
$$

If $\varepsilon$ is chosen sufficiently small, then $\frac{\varepsilon}{\pi \sin ^{2} \alpha} \leq \frac{2 \sigma^{\prime}}{2 \pi}<\frac{2 \sigma}{2 \pi}$, and the supremum in (2.11) (or in (2.12) ) is finite. By the second part of the theorem on completeness, the system $\operatorname{Exp}^{\Lambda}$ is not complete in $C\left(I_{2 \sigma^{\prime}}\right)$ and in $L^{p}\left(I_{2 \sigma^{\prime}}\right)$ after removal of one or two exponentials. Thus, the system $\operatorname{Exp}^{\Lambda}$ is not complete in $C\left(I_{d}\right)$ and in $L^{p}\left(I_{d}\right)$ for every $d>0$.

6.2. Stability for completeness and subsequences of zeros. Apparently, the following result is new.

Theorem 4. Let $\Lambda=\left\{\lambda_{k}\right\}_{k \in \mathbb{N}}$ and $\Gamma=\left\{\gamma_{k}\right\}_{k \in \mathbb{N}}$ be two sequences of points in $\mathbb{C} \backslash \mathbb{R}$ without limit points in $\mathbb{C}$. Suppose that the sequences have finite upper density and that the sums ( $c f .(1.5)$ )

$$
\sum_{k}\left|\operatorname{Im} \frac{1}{\lambda_{k}}\right|, \quad \sum_{k}\left|\operatorname{Im} \frac{1}{\gamma_{k}}\right|
$$

are finite. Let $\Lambda$ be a subsequence of zeros for $B_{\sigma}^{\infty}$. If for some $k_{0} \in \mathbb{N}$ and $s \in \mathbb{R}$ we have

$$
\sup _{t \in \mathbb{R}}\left(\sum_{k>k_{0}}\left|\operatorname{Im} \frac{1}{t-\gamma_{k}}\right|-\sum_{k>k_{0}}\left|\operatorname{Im} \frac{1}{t-\lambda_{k}}\right|\right) \leq s,
$$

then $s>-\sigma$ and $\Gamma$ is a subsequence of zeros for $B_{\sigma+s}^{\infty}$. In particular, if $s=0$, then $\Gamma$ is a subsequence of zeros for the same space $B_{\sigma}^{\infty}$.

Together with Theorem 2 this implies the following corollary in an easy and standard way.

Corollary 2. Suppose that the assumptions of Theorem 4 are fulfilled, up to the finiteness of the sums (6.11) inclusive. If (6.12) is fulfilled for some $s \in \mathbb{R}$ and $2 s<d$, then the excess exc $\Lambda$ for $C\left(\bar{I}_{d-2 s}\right)$ or $L^{p}\left(I_{d-2 s}\right)$ with $p \geq 2$ cannot be smaller than exc $\Gamma-1$, where $\operatorname{exc} \Gamma$ is the excess for $C\left(\bar{I}_{d}\right)$, respectively, $L^{p}\left(I_{d}\right)$ with the same value $p \geq 2$; also, the quantity exc $\Lambda$ for $L^{p}\left(I_{d-2 s}\right)$ with $1 \leq p<2$ cannot be smaller than exc $\Gamma-2$ for $L^{p}\left(I_{d}\right)$ with the same value of $p$.

Some commentary to this corollary is probably in order in the case where $2 s \geq d$. Then either $d=2 s, \bar{I}_{d-2 s}=\bar{I}_{0}$ is a singleton, and an arbitrary system of exponentials (even that consisting of only one function) is complete in $C\left(\bar{I}_{0}\right)$, and $I_{d-2 s}=I_{0}=\varnothing$. Also for $d<2 s$, we have $I_{d-2 s}=\bar{I}_{d-2 s}=\varnothing$, and function spaces on an empty set are empty.

Proof of Theorem 4. Since $\Lambda$ is a subsequence of zeros for $B_{\sigma}^{\infty}$ and $\operatorname{Im} \lambda_{k} \neq 0$ for all $r$, by Theorem 1 and Remark 4 we can find a constant $C \in \mathbb{R}$ such that

$$
\int_{\mathbb{R}}\left(\sum_{k} \frac{1}{\pi}\left|\operatorname{Im} \frac{1}{t-\lambda_{k}}\right|\right) \varphi(t) d t-\frac{\sigma}{\pi} \int_{\mathbb{R}} \varphi(t) d t \leq C, \quad \varphi \in R \mathcal{P}_{0}^{m} .
$$


Therefore, by (6.12), we have

$$
\int_{\mathbb{R}}\left(\sum_{k} \frac{1}{\pi}\left|\operatorname{Im} \frac{1}{t-\gamma_{k}}\right|\right) \varphi(t) d t-\frac{\sigma+s}{\pi} \int_{\mathbb{R}} \varphi(t) d t \leq C, \quad \varphi \in R \mathcal{P}_{0}^{m} .
$$

If $s \leq-\sigma$, then, denoting by $\gamma_{1}$ some point of $\Gamma$, from the last statement and the positivity of $\varphi$ we deduce that

$$
\frac{1}{\pi} \int_{\mathbb{R}}\left|\operatorname{Im} \frac{1}{t-\gamma_{1}}\right| \varphi(t) d t \leq C, \quad \varphi \in R \mathcal{P}_{0}^{m} .
$$

But the left-hand side here cannot be uniformly bounded if, for instance, the functions $\varphi$ run over the class (see examples in Subsection 4.2 above) of the functions $t \mapsto \log ^{+}(R /|t|)$, $t \in \mathbb{R}_{*}$, for $0<R \rightarrow+\infty$. Indeed, in this case the left-hand side of (6.14) cannot be smaller than

$$
\frac{1}{\pi} \int_{\mathbb{R}}\left|\operatorname{Im} \frac{1}{t-\gamma_{1}}\right| \log \frac{R}{|t|} d t=\log \frac{R}{\left|\gamma_{1}\right|} \underset{R \rightarrow+\infty}{\longrightarrow}+\infty .
$$

Thus, $s>-\sigma$, i.e., $\sigma+s>0$. Now, (6.13) and the implication 1) $\Rightarrow$ 2) in Theorem 1 show that $\Gamma$ is a nonuniqueness sequence, or a subsequence of zeros for the Bernstein space $B_{\sigma+s}^{\infty}$.

In conclusion, the authors want to express their gratitude to the referee of the first versions of this paper for discovering serious mistakes and inaccuracies in corollaries to the main results of the paper, for a series of quite useful and important remarks, and for valuable advice.

\section{REFERENCES}

[1] B. N. Khabibullin, Completeness of exponential systems and uniqueness sets, RIC Bash. Gos. Univ., Ufa, 2012. (Russian)

[2] B. Ya. Levin, Lectures on entire functions, Transl. Math. Monogr., vol. 150, Amer. Math. Soc., Providence, RI, 1996. MR1400006 (97j:30001)

[3] N. Levinson, Gap and density theorems, Amer. Math. Soc. Colloq. Publ., vol. 26, Amer. Math. Soc., New York, 1940. MR0003208 (2:180d)

[4] L. Schwartz, Approximation d'une fonction quelconque par des sommes d'exponentielles imaginaires, Ann. Fac. Sci. Toulouse (4) 6 (1943), 111-176. MR0015553(7:437d)

[5] R. M. Redheffer, Completeness of sets of complex exponential, Adv. in Math. 24 (1977), no. 1, 1-62. MR0447542(56:5852)

[6] V. P. Havin and B. Jöricke, The uncertainly principle in harmonic analysis, Ergeb. Math. Grenzg., vol. 28, Springer-Verlag, Berlin, 1994. MR.1303780 (96c:42001)

[7] A. M. Sedletskii, Fourier transforms and approximation, analytical. Methods and Spectral Functions, Gordon and Breach Sci. Publ., Amsterdam, 2000. MR1935577 (2003i:42002)

[8] Analytic Fourier transforms and exponential approximations. I, Sovrem. Mat. Fundam. Napravl. 5 (2003), 3-152; English transl., J. Math. Sci. (N.Y.) 129 (2005), no. 6, 4251-4408. MR2120868 (2005m:42011a)

[9] _ Analytic Fourier transforms and exponential approximations. II, Sovrem. Mat. Fundam. Napravl. 6 (2003), 3-162; English transl., J. Math. Sci. (N.Y.) 130 (2005), no. 6, 5083-5254. MR 2120869 (2005m:42011b)

[10] N. Makarov and A. Poltoratski, Meromorphic inner functions, Toeplitz kernels and the uncertainty principle, Perspectives in Analysis, Math. Phys. Stud., vol. 27, Springer, Berlin, 2005, pp. 185-252. MR:2215727 (2007b:47079)

[11] A. D. Baranov, Completeness and Riesz bases of reproducing kernels in model subspaces, Int. Math. Res. Not. 2006, Art. ID 81530, 2006. MR2264717 (2007i:47014)

[12] _ Model subspaces of the Hardy space (Bernstein inequalities systems of reproduction kernels, Beurling-Malliavin type theorems), Doctoral Thesis, St. Petersburg, 2011. (Russian)

[13] F. W. King, Hilbert transforms. Vol. I, Encyclopaedia Math. Appl., vol. 124, Cambridge Univ. Press, Cambridge, 2009. MR2542214 (2011c:42003)

[14] _ Hilbert transforms. Vol. II, Encyclopaedia Math. Appl., vol. 125, Cambridge Univ. Press, Cambridge, 2009. MR2542215 (2011c:42004) 
[15] J. N. Pandey, The Hilbert transform of Schwartz distributions and applications, Wiley-Intersci. Publ., 1996. MR1363489 (97e:44010)

[16] L. Schwartz, Analyse mathematique. II, Hermann, Paris, 1967. MR0226973 (37:2558b)

[17] B. N. Khabibullin, Applications in complex analysis of dual representation for functionals on vector lattices, Mathematical Forum, vol. 4, Researches on Math. Analysis Differential Equations and their Applications, Vladikavkaz, 2010, pp. 102-116. (Russian)

[18] Sets of uniqueness in spaces of entre functions of one variable, Izv. Akad. Nauk SSSR Ser. Mat. 55 (1991), no. 5, 1101-1123; English transl., Math. USSR-Izv. 39 (1992), no. 2, 1063-1084. MR $1149889(93 \mathrm{e}: 30062)$

[19] S. A. Grigoryan, Generalized analytic functions, Uspekhi Mat. Nauk 49 (1994), no. 2, 3-42; English transl., Russian Math. Surveys 49 (1994), no. 2, 1-40. MR.1283134 (95h:43006)

[20] Dual representation of superlinear functionals and its applications in the theory of functions. II, Izv. Ross. Akad. Nauk Ser. Mat. 65 (2001), no. 5, 167-190; English transl., Izv. Math. 65 (2001), no. 5, 1017-1039. MR1874358(2003b:46024)

[21] _ The distribution of zeros of entire function and the balayage, Doctoral Thesis, Khar'kov, 1993. (Russian)

[22] P. Blanchet, On removable singularities of subharmonic and plurisubharmonic functions, Complex Variables Theory Appl. 26, (1995), no. 4, 311-322. MR.1315864 (96a:32025)

[23] B. N. Khabibullin, Completeness of systems of entire functions in spaces of holomorphic functions, Mat. Zametki 66 (1999), no. 4, 603-616; English transl., Math. Notes 66 (1999), no. 3-4, 495-506. MR.1747088(2001d:32002)

[24] T. J. Ransford, Potential theory in the complex plane, Cambridge Univ. Press, Cambridge, 1995. MR.1334766 (96e:31001)

[25] J. B. Garnett, Bounded analytic functions, Pure and Appl. Math., vol. 96, Acad. Press, Inc., New York, 1981. MR628971 (83g:30037)

[26] M. Brelot, Elements dela théorie classique du potentiel, Centre Doc. Univ., Paris, 1959. MR0106366 $(21: 5099)$

[27] P. Koosis, The logarithmic integral. II, Cambridge Stud. Adv. Math., vol. 21, Cambridge Univ. Press, Cambridge, 1992. MR 1195788 (94i:30027)

[28] B. N. Khabibullin, Nonconstructive proofs of the Beurling-Malliavin theorem on the radius of completeness and nonuniqueness for entire functions, Izv. Ross. Akad. Nauk Ser. Mat. 58 (1994), no. 4, 125-148; English transl., Izv. Math. 45 (1995), no. 1, 125-218. MR1307059 (96g:30005)

[29] P. Koosis, Leçons sur le théorème de Beurling et Malliavin, Univ. de Montréal, Les Publ. CRM, Montréal, 1996. MR 1430571 (99e:42023)

Bashkir State University, ul. Zaki Validi 32, Ufa 450074, Bashkortostan, Russia

E-mail address: Khabib-Bulat@mail.ru

Received 4/FEB/ 2012

Translated by S. V. KISLYAKOV 